NBER WORKING PAPER SERIES

\title{
REAL EFFECTS OF MONETARY SHOCKS \\ IN AN ECONOMY WITH \\ SEQUENTIAL PURCHASES
}

Robert E. Lucas, Jr.

Michael Woodford

Working Paper No. 4250

\author{
NATIONAL BUREAU OF ECONOMIC RESEARCH \\ 1050 Massachusetts Avenue \\ Cambridge, MA 02138 \\ January 1993
}

We are grateful to John Kennan, Debraj Ray, Julio Rotemberg, Nancy Stokey, and Chuck Wilson for discussions, and to the National Science Foundation for research support. This paper is part of NBER's research program in Economic Fluctuations. Any opinions expressed are those of the authors and not those of the National Bureau of Economic Research. 
NBER Working Paper \#4250

January 1993

\title{
REAL EFFECTS OF MONETARY SHOCKS \\ IN AN ECONOMY WITH \\ SEQUENTIAL PURCHASES
}

\begin{abstract}
We study the effects of monetary disturbances in an economy in which sellers must deal with potential buyers in sequence, rather than being able to sell their goods in a Walrasian auction market. Because of the structure of trading assumed, the current state of demand is not revealed to sellers until after the process of sequential transactions has concluded. As a consequence, unanticipated changes in nominal spending flows induce less-than-proportional responses in nominal transaction prices, and clianges in the same direction in real output. These effects are similar to those obtained if sellers must commit themselves in advance to money prices, but do not depend upon any cost of changing prices.

We fully characterize the stationary intertemporal equilibrium of an economy subject to i.i.d. money supply shocks. We show how the ex ante distribution of monetary shocks affects sellers' pricing strategies, and hence the equilibrium relation between the money supply, the distribution of transaction prices, and the degree to which available productive capacity is utilized.
\end{abstract}

Robert E. Lucas, Jr.

Department of Economics University of Chicago

1126 E. 59th Street

Chicago, IL 60637

and NBER
Michael Woodford

Department of Economics

University of Chicago

1126 E. 59th Street

Chicago, IL 60637

and NBER 


\section{Ineroduction}

This paper is a theoretical study of the effects of monetary

disturbances in an economy where goods are exchanged for money in a certain kind of non-Walrasian market. The market is one in which sellers of goods deal with potential buyers in sequence, deciding whether to accept buyers' orders before learning how many further orders they will receive. As a result, the current state of demand is not revealed to sellers until after the process of sequential transactions has concluded. A consequence of the trading and informational structure we assume is that unanticipated changes in nominal spending flows induce less-than-proportional responses in nominal transaction prices, and as a result changes in the same direction in real output.

The idea of attributing a non-neutrality of money to the assumption that some prices are established before the current state of demand is fully revealed to all participants underlies many earlier models. In many familiar models it is simply assumed that money prices must be fixed prior to the realization of demand. Indeed, one may interpret the model of this paper as one in which producers set prices in advance, though different units of the same good may be offered for sale at different prices.

In the model of Lucas (1972), nominal prices in individual markets are set by a Walrasian mechanism, and fully reflect local demand information, but sellers in any one market do not have access to relevant information about the state of demand in other markets. In the model of that paper, buyers and sellers can negotiate any price they like at the time that an order is accepted, and all participants have full access to any relevant information in the system at that time. In our model, it is the nonWalrasian market structure and not restrictions on agents' strategies or on 
their information sets that prevents instantaneous revelation through prices of the state of aggregate demand and leads to the monetary non-neutrality.

Our model adapts a market game introduced by Prescott (1975) and Butters (1977), in which buyers search for low priced supply offers by sellers. In this game, sellers are not required to offer identical items at the same price to successive buyers, and in equilibrium do not choose to do so. As Rotemberg (1988) has stressed, this game need not involve any price commitments by sellers prior to the time at which an order is accepted. We embed this model of sequential purchases in an intertemporal model of monetary exchange where buyers are subject to cash-in-advance constraint.

Sections 2 and 3 analyze temporary equilibrium in a one-shot game with sequential purchases. This allows us to present the non-Walrasian market game while abstracting from dynamic complications. Section 4 then embeds the temporary equilibrium in a complete intertemporal monetary equilibrium, and proves the existence of a stationary equilibrium in the case of monetary shocks that are independent across periods. Section 5 discusses further the interpretation of our results, and concludes the paper.

\section{A Pricing Game with Sequential Purchases}

In this section we describe in some detail the kind of market game with sequential purchsses that we have in mind. Since our theory centers on the progressive revelation of information through the process of trading, it is important to be explicit about the exact sequence of events. We describe a simple one-shot game, an adaptation of the games introduced by Prescott (1975) and Butters (1977), in order to explain our equilibrium concept. 
There are two types of players, producers and consumers. Each producer begins with $y$ units of a single good. We may think of this as $y$ units of inventory that can be sold, or as a capacity to produce up to $y$ units. The latter interpretation is especially interesting insofar as it allows us to interpret the fluctuations in equilibrium sales derived below as fluctuations in output. Each consumer begins with one dollar. Though we use monetary language in referring to this second good in this section, the question of why people value this good will be deferred until section 4 , where it will be given as complete an answer as such a question admits. In this section, money is just a good from which some people get utility, in a way we will describe shortly.

The play proceeds as follows. First, each producer chooses a schedule of prices at which he is willing to sell successive units of the good. We might imagine that each producer places a dollar price on each unit of his endowment. But he need not, and in general will not, choose the same price for all units. Alternatively, we may suppose that a producer chooses a schedule indicating the price at which he is willing to sell a unit of his remaining inventory (or commit a unit of his remaining uncomitted capacity) as a function of the number of units already sold.

Second, each consumer recelves a dollar transfer that transforms his money holdings from 1 to $\theta$, where $\theta$ is a positive-valued random variable with the probability measure $\Phi$ on an interval $\theta-[\theta, \bar{\theta}] \subset \mathbf{R}_{+}$. The distribution is known to producers at the time they choose their supply schedule, but the realization $\theta$ is not. Third, consumers use some or all of their balances $\theta$ to purchase goods. It is assumed that 
consumers can costlessly search across producers for the lowest-priced supply offer.

In this section and the next, we will develop a definition of a symetric equilibrium of a game that makes this search process explicit and characterize its equilibrium. To anticlpate this development, an equilibrium will involve two functions $p(\theta)$ and $c(\theta)$ of the money supply shock $\theta$, where $\mathrm{P}(\theta)$ is the highest, or marginal, price at which goods are purchased and $c(\theta)$ is the total consumption of each consumer. Consumers will be assumed to have a marginal utllity of unspent cash that is independent of $\theta$, so they will purchase goods up to the point at which the marginal utillty of current consumption spending just equels this marginal return to cash holding, or at which they exhaust thelr cash. Producers will be assumed to maximize expected return, so they w1ll offer goods for sale in such a way that the price of each unit times the probability that that unit will be sold is equated across all prices at which goods are offered. By developing the implications of these two simple principles, we will construct an equilibrium in which an array of goods are offered at different prices, wich low priced units actually sold for almost all values of $\theta$ and high priced units sold only at very high $\theta$ values.

To formulate a definition of the equilibrium of this game, we need a description of the strategies avallable to producers and consumers, and of the process that matches consumers to the differently priced goods made available by producers. In this section, we sketch such a description for a game with a continum of producers and consumers, and with a countable set 9 of possible prices, and then use this game to suggest a definition of an equilibrium in which any real number can serve as a price. This definition, on which all of the results in the paper are based, appears at the end of 
this section. In Section 3, we use the first-order and market clearing conditions to show that there is a unique symetric equilibrium of this game and to characterize this equilibrium.

We assume a continum of length one for both player types. For our initial example, we require all transaction prices to lie in a countable set $P=\left(p_{1}, P_{2}, \ldots\right)$, where $0<p_{1}<p_{2}<\ldots$ Since consumers can costlessly search for the lowest-price supply offers, each would like to acquire whatever goods he is consuming at the lowest price $p_{1}$, and none at any higher price. If this is possible (if demand at $p_{1}$ with no consumer rationed is less than or equal to the total quantity of goods offered for sale at $p_{1}$ ) we will call this an equilibrium, but in general it is clear that some non-price rationing scheme will be needed. We imagine that this rationing is done (by an auctioneer, if you like) as follows.

Beginning with the lowest price $p_{1}$, each consumer $i$ submits a buy order $x_{1 i}$, subject to the constraint $p_{1} x_{1 i} \leq \theta$, and each producer $j$ submits a supply offer $y_{1 f}$, subject to the constraint $y_{1 j} \leq y$. If the aggregate of these orders, $D_{1}-\int x_{11} d i$, is less than or equal to aggregate supply, $s_{1}-\int y_{1 j} d f$, the game ends at stage 1.1 Each consumer $i$ receives $x_{11}$ units of the good, and foregoes $p_{1} x_{11}$ of his cash. Each producer sells the fraction $D_{1} / s_{1}$ of the goods $y_{1 f}$ that he has offered at the lowest price, and all his remaining goods go unsold. If $D_{1}>s_{1}$, each producer sells all the goods he has offered at $p_{1}$, and each consumer $i$ receives $c_{11}-\left(s_{1} / D_{1}\right) x_{11}$, paying $p_{1}$ for each unit. In this case, the game proceeds to a stage 2 .

In any stage $k \geq 2$, if $1 t$ is reached, consumer 1 submits a buy order $x_{k i}$, subject to the constraint imposed by the cash holdings remaining from earlier stages: 


$$
P_{k} x_{k i} \leq \theta-\sum_{s=1}^{k-1} p_{s} c_{s i} .
$$

Froducer $j$ submits a supply offer $y_{k i}$, not exceeding his inventory remaining from earlier stages:

$$
y_{k j} \leq y-\sum_{s=1}^{k-1} y_{s j} .
$$

If the aggregate of these orders, $D_{k}-\int x_{k i} d i$, is less than or equal to aggregate supply, $s_{k}=\int y_{k j} d j$, the game ends at stage $k$. Each consumer $i$ receives $x_{k i}$, and foregoes $p_{k} x_{k i}$ of his remaining cash. Each producer $j$ sells the fraction $D_{k} / S_{k}$ of the goods $y_{k j}$ he has offered at the price $P_{k}$, and all his remaining goods go unsold. If $D_{k}>S_{k}$, each producer sells all the goods he has offered at $P_{k}$, and each consumer $i$ receives $c_{k i}$ - $\left(S_{k} / D_{k}\right) x_{k i}$, paying $P_{k}$ for each unit. In this case, the game proceeds to stage $k+1$.

So the process continues, with the game ending whenever $D_{n} \leq S_{n}$. Each consumer's payoff is given by $U\left(c_{1}\right)+\alpha w_{1}$, where $c_{1}-\Sigma_{s=1}^{n} c_{s i}$ is his total consumption (here $c_{n i}=x_{n i}$ ), $w_{1}-\theta-\Sigma_{s-1}^{n} p_{s} c_{s i}$ is his unspent cash, $\alpha$ is a positive parameter, and $U(\cdot)$ is a strictly increasing function. The reason for assigning a value to unspent cash will be clarified when we proceed to the multi-period game of Section 4. Each producer's payoff is given by the dollar value of his sales revenues, summed over all $n$ stages. Producers obtain no payoff from unsold inventory or unused capacity. Each type of player seeks to maximize expected payoff. The reason why producers seek to maximize expected revenues is also clarified in Section 4. 
Information is revealed as follows. At the beginning of stage 1, each consumer learns the value of $\theta$, but producers do not. At the end of each stage, each producer learns the number of goods that he has been able to sell, each consumer learns the number of goods that he has been able to buy, and both types learn whether the game proceeds to another stage or not. In fact, without changing the equilibrium concept we may assume that all sales are publ1s. What is important is that the aggregate orders $D_{k}$ are not revealed, but only the purchases that actually occur. This assumption is intended to capture the idea that producers learn about the state of aggregate demand only through the number of sales that have aleady been made.

A strategy for consumer $i$ in this game specifies his buy order $x_{k i}$ at each stage $k-1,2, \ldots$ as a function of his information at that stage Thus the consumer chooses a sequence of the form

$$
x_{k i}\left(\theta, c_{1 i}, \ldots, c_{k-1, i}\right)
$$

each term of which satisfies (2.1) for all values of the arguments. We uight, if we like, add as arguments of the $x_{k i}$ the complete history of sales that have occurred in the first k-1 stages of the game. However, given the strategies of all players other than consumer $i$, this history is a function of $\theta$, and so it suffices (in defining a Nash equilibrium) to allow consumer 1 to choose a function of the ford written above. ${ }^{2}$

A producer's information is always the same when he reaches stage $k$ : he knows that he has sold all the units that he offered for sale at prices $p_{1}, \ldots, p_{k-1}$, and he knows nothing else about the current state. We ulght, 
symetrically with our notation for consumers, write producer j's strategy as a sequence of functions

$$
y_{k j}\left(z_{1 j}, \ldots, z_{k-1, j}\right)
$$

where $z_{s j}$ represents $j$ 's sales in stage $s$. However, payoffs will depend only on the sequence of quantities

$$
y_{k j}-y_{k j}\left(y_{1 j}, \ldots, y_{k-1, j}\right)
$$

Hence we may describe a producer's strategy as simply a measure $\pi$ on the set $P$ satisfying: ${ }^{3}$

$$
\pi(P) \leq y .
$$

Let $\bar{\pi}_{j}$ be the measure chosen by producer $j$. The strategies of the several producers together determine an aggregate measure $\pi-\int \pi_{j} d j$. Aggregate supply at each price is then given by $s_{k}=\pi\left(P_{k}\right)$.

Note that the strategles available to producers are the same as in a game in which each producer must place a price tag, in dollar terms, on each unit of his endowment, prior to the realization of the shock $\theta$, and must then sell each unit at the price indicated in advance if a consumer offers to buy it. Hence it is not surprising that variations in $\theta$ have real effects in this model that are similar to those in an economy in which money prices are posted in advance, of the kind studied by the authors cited in the introduction. Yet here the result does not depend upon any inability of producers to determine freely the price at which they are willing to sell at 
the time that an order is accepted. At each stage $k$, the producer is free to determine the number of units (if any) that he is willing to supply at the price $P_{k}$, and is forced to accept no orders in excess of that number.

The producer's strategy $\pi_{j}$ is independent of $\theta$, because producers do not observe $\theta$ prior to accepting orders. Consumers instead observe $\theta$, and so $\theta$ is an argument of each function $x_{k 1}(\cdot)$. Given the strategy $\pi_{j}$ for each producer, and the strategy $\left(x_{11}(\theta), x_{21}\left(\theta, c_{11}\right), \ldots\right)$ for each consumer, the process we have described determines a unique final stage $n(\theta)$, as we 11 as a unique sequence $\left(c_{1 i}(\theta), c_{21}(\theta), \ldots, c_{n i}(\theta)\right)$ for every consumer, a dollar expenditure for every consumer, a total quantity sold and a total dollar revenue for each producer, all for each possible value of $\theta$. The payoffs to each player are thus deterwined as a function of $\theta$.

In the game whose rules we have just described, the payoff to any player depends only on his strategy choice and the aggregate quantities offered and bid for at the varlous prices. An individual player's effect on these aggregates is negligible. ${ }^{4}$ In particular, an individual producer's choice of $\pi_{f}$ has no effect upon which stage $n$ (as a function of $\theta$ ) is the final stage, or upon the fraction of goods offered for sale at the price $p_{n}$ that are sold (also a function of $\theta$ ). He therefore takes as given the final stage $n(\theta)$ and the fraction of goods unsold at that stage, $u(\theta)$ $\left(S_{n}-D_{n}\right) / S_{n}$, and chooses $\pi_{j}$ to maximize the expected value of

$$
\sum_{s=1}^{n(\theta)} \pi_{j}\left(p_{s}\right) p_{s}-u(\theta) \pi_{j}\left(p_{n(\theta)}\right) p_{n}(\theta)
$$

subject to the constraint (2.2). The expectation is taken over possible realizations of $\theta$, using the measure $\Phi$. 
An individual consumer similarly takes as given the fraction of the goods bid for at the price $p_{k}$ in stage $k$ that he will be able to buy, as this fraction is unaffected by his own strategy. He will therefore bid for as many units as he can (given the constraints (2.2)) at each stage prior to the last stage in which he bids for any positive quantity.

In what follows, we focus attention on a specific kind of syometric equiliorium of this game, in which all consumers play the same strategy. Furthermore, we consider only symetric equilbria with the property that if a stage $k$ is reached such that consumers would wish to purchase no more than is supplied at the price $p_{k}$, were they allowed to buy an unlimited quantity at that price, then in such a stage they would buy only the number of units they actually wish to consume. This latter restriction rules out an equilibrium in which all consumers bid for, say, 25 more units in stage $k$ than they actually wish to buy, because they know that excess demand will necessitate their being rationed to only 80 of their bids. There is no loss of generality in ignoring such equilibria, since there will always be an equivalent equilibrium (in the sense that the same allocations of goods and money result) in which consumers bid for only the number of units desired, and hence are not rationed.

We observe that in any such equilibrium each consumer's optimal strategy is to bid all of his remaining cash in every stage prior to $n$ - the first stage in which consumers are not rationed, and the last stage reached in the game. For since consumers are rationed in stage $\mathrm{n}-1$, they must bid for a positive quantity in that stage; hence they bid all of their avallable cash in every stage $k<n-1$. Moreover, in stage $n-1$, they also bid all of their available cash, because each would like to purchase more than his share of what is supplied at the price $\mathrm{P}_{\mathrm{n}-1}$; otherwise, by our assumption 
in the preceding paragraph, they would not be rationed in stage n-l. Hence If the terminal stage of the game is $n(\theta)$, the equilibrium strategy of consumers is completely described by the quanticy $c_{n}(\theta)$ bid for (and, under the convention adopted above, received) in that final stage. Equivalently, one may describe a consumer's strategy in a symetric equilibrium by $n(\theta)$ and $c(\theta)$, the total units purchased in all stages, since the quantity purchased in each stage $k<n$ must equal the supply $\pi\left(\mathrm{P}_{\mathrm{k}}\right)$.

Now consider the choices avallable to a single non-atomic consumer, who takes as given the identical strategies chosen by all other consumers, as just described, and the aggregate measure of goods offered at various prices by producers. Since all other consumers bid all of their remaining cash at each stage $k<n$, the most an Individual consumer can obtain at that stage, assuming he has obtained the maximum amount at all earlier stages, is the amount $c_{k}=\pi\left(p_{k}\right)$ purchased by the other consumers. On the other hand, in stage $n$, the consumer can obtain any amount he bids for, since consumers are not rationed at that stage. This budget set can be conveniently described as follows. Let the supply function $S_{\pi}: R_{+} \rightarrow \bar{P}-P U$ $(+\infty)$ be defined by:

$$
s_{\pi}(c)=\inf \left(p_{n} \in P \mid \pi\left(\left(p_{1}, \ldots, P_{n}\right)\right) \geq c\right)
$$

(When the set in brackets is empty, the infimum is defined to be to.) Note that $S_{\pi}$ is an alternative description of the measure $\pi-a$ kind of cdf of $\pi$. If a total quantity $c$ is purchased and the lowest-priced goods are purchased first, $S_{\pi}(c)$ represents the price of the last unit purchased. 
Then if his initial cash is $\theta$ and the terminal stage is $n$, a consumer can bid for any amount $c$ satisfying:

$$
\int_{0}^{c} \min \left(S_{\pi}(x), p_{n}\right) d x \leq \theta
$$

and his remaining cash will be the amount by which $\theta$ exceeds the left hand side. The consumer's optimal bid will then be:

$$
c(\theta ; n)-\arg \max _{c}\left(U(c)+\alpha\left[\theta-\int_{0}^{c} \min \left(s_{\pi}(x), p_{n}\right] d x\right)\right.
$$

where the maximization is subject to (2.5a).

A symetric equilibrium then involves an $n(\theta)$ and $c(\theta)$ such that

$$
n(\theta)-\inf \left(n \mid c(\theta ; n) \leq \pi\left(\left(p_{1}, \ldots, p_{n}\right)\right)\right.
$$

$$
c(\theta)-c(\theta ; n(\theta)) \text {. }
$$

(Again, we define inf $\emptyset=+\infty$, and $P_{n}-+\infty$ if $n-+\infty$ )

Conditions (2.6) define equilibrium play by consumers, given the aggregate supply measure $\pi$ of producers. On the other hand, the obfective function (2.3) of an individual, non-atomic producer is defined by the function $n(\theta)$ describing consumers' strategies and the function

$$
u(\theta)-\left[\pi\left(P_{n}\right) \cdot c_{n}(\theta)\right] / \pi\left(P_{n}\right)
$$


that is implied by consumers' strategies and the aggregate supply measure chosen by other producers. A symetric Nash equilibrium for the continuum game is thus a measure $\pi$ on $P$, and measurable functions $n: \theta \rightarrow N, u: \theta \rightarrow$ $[0,1]$, and $c: \theta \rightarrow \mathbf{R}_{+}$, such that $(c, n)$ satisfy $(2,6)$, given $\pi ; u$ satisfies (2.7), given $(c, \pi)$; and $\pi$ maximizes the expected value of $(2.3)$, subject to the constraint $(2,2)$, given $(n, u)$.

We next adapt this definition of equilibrium to the case in which the set of possible prices is the entire nonnegative real line. We proceed directly from the above characterization of a symmetric equilibrium, rather than introducing notation for the underlying strategies and game form. An equilibrium will now consist of a measure $\pi$ in the set $M$ of all measures on the Borel sets of $\mathbf{R}_{+}$, and Borel-measurable functions $p, u$, and $c$ on 8.

As before, we imagine a game proceeding through prices from 0 on up to some terminal price $p(\theta)$ that depends on the state of demand $\theta$. At each price below $p(\theta)$, consumers bid all of their cash and are rationed to receive equal shares of the goods on offer at that price. Producers sell all of the goods offered at that price. At the terminal price $p(\theta)$, consumers are not rationed, while producers can sell a fraction $1-u(\theta)$ of the goods they have offered at $p(\theta)$. In a symetric equilibrium with a continum of both player types, consumers take as given the terminal price $p(\theta)$ and their rations at prices lower than $p(\theta)$, determined by the supply distribution $\pi$ chosen by producers. Their actions determine the number $c(\theta)$ of goods purchased. Producers take as given the price $p(\theta)$ and the rationing function $u(\theta)$. Their actions determine the supply distribution $\pi$. 
Each producer's problem in this game is to choose a measure $\pi \in M$ that satisfies $(2.2)$ so as to maximize $\mathbb{E}[R(\theta)]-\int R(\theta) \Phi(d \theta)$, where $R(\theta)$ is his dollar revenues in state $\theta$, given by:

$$
R(\theta)=\int_{0}^{P(\theta)} z \pi(d z)-u(\theta) P(\theta) \pi((P(\theta)))
$$

Here (2.8) is an obvious generalization of (2.3).

Each consumer's problem can similarly be described in a way that difectly generalizes (2.4)-(2.6). We can again define a supply function $S_{\pi}: \mathbf{R}_{+} \rightarrow \overline{\mathbf{R}}_{+}-\mathbf{R}_{+} \cup(+\infty)$ by:

$$
S_{\pi}(c)=\inf (p \geq 0 \mid \pi([0, p]) \geq c)
$$

Note that $S_{\pi}$ is a non-decreasing, left-continuous function (see Figure 1 in the next section). Its flats correspond to mass points of the supply distribution $\pi$, while its jumps correspond to price intervals on which $\pi$ offers no goods.

The consumer's problem, at each stage $p$ of the game, is to choose $c$ so as to maximize

$$
\mathrm{U}(c)+a\left[\theta-\int_{0}^{c} \min \left[\mathrm{S}_{\pi}(\mathrm{x}), \mathrm{p}\right] \mathrm{dx}\right]
$$

subject to 
$(2.10 b)$

$$
\theta \geq \int_{0}^{c} \min \left[s_{\pi}(x), p\right] d x .
$$

Let $c(\theta ; \mathrm{p})$ be the solution to this problem.

We assume that $U$ is continuously differentiable on $\mathbf{R}_{+}$, strictly increasing and strictly concave, with lim $U^{\prime}(c)=+\infty$. The integral in $c \rightarrow 0$

$(2.10)$ is well-defined for $a l l c>0$ and $p \cdot \in \overline{\mathbf{R}}_{+}$. It is a continuous, non-decreasing function of $c$, so the constraint set is a closed interval, bounded if $p>0$. The function $(2.10 \mathrm{a})$ is continuous and strictly concave. Hence if $p>0$ there is a unique maximum of $(2.10 \mathrm{a})$. If $p-0$, the function (2.10a) is increasing for all $c$ and we define $c(\theta ; 0)-+\infty$. Thus $c(\theta ; p)$ is well defined on all of $\mathbf{R}_{+} \times \overline{\mathbf{R}}_{+}$. It is also easily shown that $c(\theta ; p)$ is non-increasing as a function of $p$, and continuous in $p$ for all $\mathrm{P}>0$.

The direct generalization of (2.6) to the case of a continum of prices is thus:

$$
\begin{aligned}
& p(\theta)=\inf (p \mid c(\theta ; p) \leq \pi([0, p])), \\
& c(\theta)=\min [c(\theta ; p(\theta)), \pi([0, p(\theta)])] .
\end{aligned}
$$

where again we define inf $\phi-+\infty$. The second alternative in $(2.11 \mathrm{~b})$ applies if $c(\theta ; p) \leq \pi([0, p])$ for all $p>p(\theta)$, but nonetheless $c(\theta ; p)>$ $\pi([0, p])$. Because of the continuity of $c(\theta ; p)$ in $p,(12.11 b)$ implies

$$
c(\theta)-c(\theta ; p(\theta)) \text { if } p(\theta)>0 \text {, }
$$




$$
c(\theta)-\pi(10)) \text { if } p(\theta)-0 \text {. }
$$

The corresponding generalization of (2.7) is

$$
u(\theta)-\frac{\pi(10, R(\theta)])-c(\theta)}{\pi(\{p(\theta)\})} \text { if } \pi((p(\theta)\})>0 \text {, }
$$

$$
u(\theta)-0
$$$$
\text { if } \pi((p(\theta)))-0 \text {. }
$$

We can then define an equilibrium for the continuum game as follows:

Definition A temporary equilibrium (TE) is a measure $\pi \in M$, a function $S_{\pi}: R_{+} \rightarrow \bar{R}_{+}$defined in terms of $\pi$ by (2.9), and a triple of measurable functions $p: \theta \rightarrow \overline{\mathbf{R}}_{+}, u: \theta \rightarrow[0,1]$, and $c: \theta \rightarrow \mathbf{R}_{+}$, such that $(1)$ given $(p, u)$, $\pi$ maximizes the expected value of (2.8), subject to (2.2); (1i) for each $\theta$ $\epsilon \theta$ and given $\pi,(p, c)$ satisfy (2.11); and (iii) for each $\theta \in \theta$ and given $(\pi, p, c), u$ satisfies $(2.12)$.

We call this equilibrium temporary because it represents equilibrium play in a single period, taking as given the expectations about the future value of money reflected in the parameter $\alpha>0$. In Section 4 we will define and characterize an intertemporal equilibrium in which the equilibrium value of $\alpha$ is determined.

\section{Characterization of Temporary Equilibria}


In this section, we use the consumer's problem and the producer's problem in turn to characterize the temporary equilibria defined at the close of Section 2. We first use the first-order conditions for the consumer's problem in a TE to show that given any producer behavior $\left(\pi, S_{\pi}\right)$ there is a shock value $\hat{\theta}>0$ with the property than consumers spend all their cash when $\theta \leq \hat{\theta}$, and spend $\hat{\theta}$ when $\theta \geq \hat{\theta}$. We characterize $\hat{\theta}$, and then (in (3.1) and (3.2)) characterize $c(\theta)$ and $p(\theta)$ in terms of $\hat{\theta}$ and $S_{\pi}$.

The Kuhn-Tucker conditions for the problem "maximize (2.10a) subject to $(2.10 b)^{n}$, together with (2.11b), imply that for all such that $p(\theta)>$ 0 , the equilibrium values of $c(\theta)$ and $p(\theta)$ must satisfy

$$
U \cdot[c(\theta)] \geq \alpha S_{\pi}(c(\theta)) \text { and } \int_{0}^{c(\theta)} S_{\pi}(x) d x \leq \theta \text {, }
$$

and $e$ ither

$$
\int_{0}^{c(\theta)} s_{\pi}(x) d x=\theta
$$

or

$$
\alpha S_{\pi}(c(\theta)) \leq U^{\prime}[c(\theta)] \leq \alpha S_{\pi}^{+}(c(\theta))=\alpha \underset{c+c(\theta)}{ } S_{\pi}(c) .
$$

Now Erom previously discussed properties of $U$ and $S_{\pi}$, there is exactly one $\hat{c} \in\left[0, \pi\left(R_{+}\right)\right]$such that $s_{\pi}(\hat{c}) \leq a^{-1} U^{\prime}(\hat{c}) \leq s_{\pi}^{+}(\hat{c})$ (see Figure 1). Hence when $P(\theta)>0$, either 
$c(\theta)=\hat{c}$ and $\int_{0}^{\hat{c}} s_{\pi}(x) d x \leq \theta$

오

$c(\theta) \leq \hat{c}$ and $\int_{0}^{c(\theta)} s_{\pi}(x) d x-\theta$.

[INSERT FIGURE I ABOUT HERE]

One observes furthermore that for any $\theta \in \theta$, there exists $p>0$ such that $\mathrm{c}(\theta ; \mathrm{p})>\pi((0))$. Hence (2.11a) implies that $\mathrm{p}(\theta)>0$ for all $\theta \in \theta$, and the above results apply in any $\mathrm{TE}$.

Define $F(c)=\int_{0}^{c} s_{\pi}(x) d x$, let $F^{-1}$ denote its inverse when the argument of the function is positive and no greater than $\pi\left(\mathbf{R}_{+}\right)$, and define $\left.F^{-1}(0)-\sup \left\{c \geq 0 \mid \int_{0}^{c} s_{\pi}(x) d x-0\right\}-\pi(10)\right)$. Then it follows that equilibrium consunption is given by:

$$
\begin{array}{ll}
c(\theta)=F^{-1}(\theta) & \text { if } \theta \leq \hat{\theta}-F(\hat{c}) \\
c(\theta)=\hat{c} & \text { if } \theta \geq \hat{\theta} .
\end{array}
$$

Note that $c(\theta)$ is a continuous, non-decreasing, concave, nonnegativevalued function. If $\pi\left(\mathbf{R}_{+}\right)>0, \hat{c}>0$ and $c(\theta)$ is positive for all $\theta \in$ $\theta$ and strictly increasing on $(\theta, \hat{\theta})$.

Given the behavior of equilibrium quantities, the behavior of prices is imediate. 


$$
\begin{aligned}
& p(\theta)-s_{\pi}[c(\theta)] \text { if } \theta \leq \hat{\theta} \\
& p(\theta)-\alpha^{-1} U^{\prime}(\hat{c}) \text { if } \theta>\hat{\theta} .
\end{aligned}
$$

Note that $p(\theta)$ is a non-decreasing function, constant when $\theta>\hat{\theta}$, and positive for all $\theta \in \theta$.

We next study the seller's problem. We show that all goods are offered for sale at positive prices $((3,3))$, that sellers are never rationed in equilibrium $((3.7))$, and that expected returns per unit of goods, the product $p(\tilde{\theta}) \operatorname{Prob}(\theta \geq \bar{\theta})$, are equated to a constant value $\lambda$ for all $\bar{\theta} \leq \hat{\theta}$ $((3.8))$. The value of this constant $\lambda$ is given in (3.9).

We first verify

$$
\pi\left(\mathbf{R}_{+}\right)-y \text { and } \pi((0))=0 .
$$

For any $q \geq 0$, let $\Lambda(q)-q\left[\operatorname{Prob}(p(\theta) \geq q)-\int u(\theta) I((\theta|p(\theta)-q|) \Phi(d \theta)]\right.$ (where $I(A)$ is the indicator function for the set $A)$. Thus $\Lambda(q)$ is the expected revenues per unit of goods priced at $q$. Note that for any $0<q$ $<p(q), \Lambda(q)-q>0$. Then if $\pi$ places an aton at the price zero, or if $\pi\left(R_{+}\right)<y$, revenues can be increased by adding goods at the price $q$, caking away the atom at zero or else increasing the total measure $\pi\left(\mathbf{R}_{+}\right)$to $y$. This proves $(3,3)$.

It follows from (3.3) that $\hat{c}-y$. For (3.1) and (3.2) imply that $\Lambda(q)-0$ for all $q>s_{\pi}(\hat{c})$. Hence $\pi$ cannot maximize expected revenues unless $\pi\left(\left[0, s_{\pi}(\hat{c})\right]\right)-y$. But the definition of $s_{\pi}$ implies that $\pi\left(\left[0, s_{\pi}(\hat{c})\right]\right)=\hat{c}$, and so that $\hat{c}-y$. 
Let the maximized value of sellers' objectfve function be $\lambda y$. We next verify that:

(3.4) $0<\lambda<\infty, \Lambda(q) \leq \lambda$ for all $q \geq 0$, and $\Lambda(\bar{q})-\lambda$ for some $\tilde{q}>0$.

We have fust seen that $\Lambda(q)>0$ for some $q$. Hence $\lambda>0$. On the other hand, (3.1) and (3.2) Imply that $p(\theta) \leq \alpha^{-1} U^{\prime}(\hat{c})-\alpha^{-1} U^{\prime}(y)$ for all $\theta$, so that $\Lambda(q) \leq a^{-1} U^{\prime}(y)$ for all $q$, and thus $\lambda y-\int \Lambda(p) \pi(d p) \leq a^{-1} U^{\prime}(y) y$ $<\infty$. Hence $\lambda<\infty$. If $\Lambda(q)>\lambda$ for any $q$, expected revenue could be made to exceed $\lambda y$ by choosing $\pi$ such that $\pi((q))-y$. Hence $\Lambda(q) \leq \lambda$ for all $q$. Finally, the facts that $\int \Lambda(p) \pi(d p)-\lambda y$ and $\int \pi(d p)-y$ imply that $\Lambda(\tilde{q})-\lambda$ for some $\bar{q}$, which must be positive. This proves (3.4).

In fact, the last two statements in (3.4) can be strengthened:

$$
\Lambda(q)=\lambda \text { for all } q \in \operatorname{supp} \pi .
$$

The statement "q $\in$ supp $\pi^{*}$ means either that $\pi$ places an atom at $q$, or that $\pi\left(N_{r}\right)>0$ for every right nelghborhood $N_{r}$ of $q$, or that $\pi\left(N_{l}\right)>0$ for every left neighborhood $N_{2}$ of $q$. If $\pi((q))>0$ and $\Lambda(q)<\lambda$, revenues could be increased by moving the atom from $q$ to the point $\bar{q} 0$ : (3.4). Hence $\Lambda(q)-\lambda$ for any atom $q$ of $\pi$. Suppose, then, that $\pi(|q|)-0$. Then $u(\theta)=0$ for all $(\theta \mid p(\theta)-q\}$, and so $\Lambda(q)-$ $q \operatorname{Prob}(p(\theta) \geq q)$. Note that 1 im $\operatorname{prob}(p(\theta) \geq p)-q \operatorname{Prob}(p(\theta) \geq q) \geq$ lim $\operatorname{prob}(p(\theta) \geq p)$. Hence $\Lambda(q)<\lambda$ 1mplies $q \operatorname{Prob}(p(\theta) \geq q)<\lambda$, which $p \downarrow q$

in turn implies that there exists both a left nelghborhood $N_{l}$ of $q$ (If $q$ 
$>0$ ) and a right neighborhood $N_{r}$ of $q$ (if $q \geq 0$ ) such that $\operatorname{pProb}(p(\theta) \geq$ $p)<\lambda$ for all $p \in N_{l} \cup N_{5}$. Hence $\Lambda(p)<\lambda$ for all $p \in N_{\ell} \cup N_{5}$. In fact, we can choose these sets so that $\Lambda(p)<\bar{\lambda}$ for all $p \in N_{\ell} \cup N_{r}$, for some $\bar{\lambda}<\lambda$. Since either $\pi\left(N_{l}\right)$ or $\pi\left(N_{r}\right)$ must be positive, it follows that expected revenues can be increased by moving all goods with prices in these sets to the price $\bar{q}$. This verifies (3.5).

Note that (3.5) implies that supp $\pi$ is bounded away from zero, since $q \geq \lambda>0$ for all $q \in \operatorname{supp} \pi$.

The next result together with (3.5) implies that sellers are never rationed in equilibrium: $q \operatorname{Prob}(p(\theta) \geq q)-\lambda$ for all $q \in \operatorname{supp} \pi$.

If $\pi((q))-0$, then $(3,6)$ is immediate from (3.5) and the definition of $A$. Suppose instead that $\pi(\{q))>0$. In any event, $q \operatorname{Prob}(p(\theta) \geq q) \geq \Lambda(q)-$ $\lambda$. If $q \operatorname{Prob}(p(\theta) \geq q)>\lambda$, then by the argument used to prove (3.5) there exists a left neighborhood $N_{l}$ of $q$ such that $p \operatorname{Prob}(p(\theta) \geq p)>\lambda$ for all $p \in N_{\ell}$. (Recall that $q \in \operatorname{supp} \pi$ implies that $q>0$.) since $\pi$ cannot place atoms at all points in the open set $N_{L}$, there exists some $p \in$ $N_{\ell}$ with $\pi((p))=0$. This contradiction proves $(3.6)$. It follows from (3.6) that

$$
\int u(\theta) I((\theta \mid p(\theta)-q)) \Phi(d \theta)-0
$$

for all $q \in$ supp $\pi$. Hence sellers are never rationed in equilibrium. Next, we have: 


$$
\mathrm{P}(\bar{\theta}) \operatorname{Prob}(\theta \geq \bar{\theta})-\lambda \text { for all } \&<\tilde{\theta} \leq \hat{\theta} .
$$

From (3.2), $\bar{\theta} \leq \hat{\theta}$ implies $p(\bar{\theta})-s_{\pi}[c(\bar{\theta})]$, and since $c(\theta)>0, s_{\pi}[c(\theta)]$ E supp $\pi$. Hence by $(3.6), p(\bar{\theta}) \operatorname{Prob}(p(\theta) \geq p(\bar{\theta}))-\lambda$. Since $p(\cdot)$ is non-decreasing, $\operatorname{Frob}(\theta \geq \bar{\theta}) \leq \operatorname{Prob}(\mathrm{p}(\theta) \geq \mathrm{p}(\bar{\theta}))$, so $\mathrm{p}(\bar{\theta}) \operatorname{Prob}(\theta \geq \bar{\theta}) \leq \lambda$. Suppose $\operatorname{Prob}(\theta \geq \tilde{\theta})<\operatorname{Frob}(P(\theta) \geq P(\bar{\theta}))$. Then there is a set $A \subset \theta$ with $\Phi(A)>0$ such that $\theta<\bar{\theta}$ and $\mathrm{p}(\theta)-\mathrm{p}(\tilde{\theta})$ for all $\theta \in A$.

Furthermore, A and $\theta^{\prime}$ can be chosen so that $\theta \leq \theta^{\prime}<\bar{\theta}$ for all $\theta \in A$. From (3.1), $\theta \leq \theta^{\prime}<\bar{\theta} \leq \hat{\theta}$ implies $c(\theta) \leq c\left(\theta^{\prime}\right)<c(\bar{\theta})$ for all $\theta \in A$. Since $p(\theta)=p(\bar{\theta}),(3.2)$ implies $\pi([0, p(\theta)])-c(\theta)-\pi([0, p(\bar{\theta})])-c(\theta) \geq$ $c(\bar{\theta}) \cdot c(\theta)>0$ for all $\theta \in A$. This fact together with the fact that $\pi((\mathrm{p}(\theta)))=\pi((\mathrm{P}(\bar{\theta}))) \geq \Phi(\mathrm{A})>0$ Implies that $u(\theta)$ is bounded away from zero on A. This contradicts (3.7) and proves (3.8).

We have now characterized the function $p(\theta)$ up to an unknown positive constant $\lambda$, given the distribution $\Phi$. Equation (3.8) gives $p(\theta)$ on the interval $[\hat{\theta}, \hat{\theta}]$. Equation $(3.2 b)$ and the fact that $\hat{c}-y$ implies $p(\theta)=\alpha^{-1} U^{\prime}(y)$ when $\theta>\hat{\theta}$. Moreover, $\lambda$ must satisfy:

$$
\lambda[\operatorname{Prob}(\theta \geq \hat{\theta})]^{-1} \leq \alpha^{-1} \mathcal{U}^{\prime}(y) \leq \lambda[\operatorname{Prob}(\theta>\hat{\theta})]^{-1} .
$$

The first inequality follows from (3.2), (3.8), and the fact $\hat{c}-y$. If $\alpha^{-1} U^{\prime}(y)=p(\hat{\theta})$, then the upper bound obviously holds. Suppose instead that $\alpha^{-1} U^{\prime}(y)>p(\hat{\theta})$. Then $\Lambda\left(\alpha^{-1} U^{\prime}(y)\right)=\alpha^{-1} U^{\prime}(y) \operatorname{Prob}\left(p(\hat{\theta}) \geq a^{-1} U^{\prime}(y)\right)-$ $a^{-1} J^{\prime}(y) \operatorname{Prob}(\theta>\hat{\theta})$, so the upper bound follows from the requirement that $\Lambda\left(a^{-1} U^{\prime}(y)\right) \leq \lambda$.

With the problems of both consumers and firms thus characterized, we turn to the characterization of temporary equilibria. For this purpose, it 
Is conventent to use the shock distribution to define a function $G$ on $\mathbf{R}_{+}$as follows:

$$
G(\bar{\theta})=\int_{0}^{\tilde{\theta}} \operatorname{Prob}\left(\theta \geq \theta^{\prime}\right) \mathrm{d} \theta^{\prime}, \text { all } \bar{\theta} \geq 0 \text {. }
$$

$G$ is continuous, increasing, and concave, with $G(0)=0$ and $G(\bar{\theta})-E(\theta)$. Because $G$ is concave, we can define its subdifferential $a G(\bar{\theta})$ :

$$
\partial G(\bar{\theta})-(\boldsymbol{B} \in \mathbf{R} \mid G(\theta)-G(\bar{\theta}) \leq \mathbf{g}(\theta-\bar{\theta}) \text { for } \mathbf{a l l} \theta \geq 0) \text {. }
$$

Then for all $\partial \geq 0, \partial G(\nexists)$ is a non-empty, closed, convex set, and the correspondence $\partial G$ is non-increasing and upper-hemi-continuous. Moreover, $\partial G(0)-[1, \infty)$ and $\operatorname{lnf} \partial G(\bar{\theta})-0$.

Proposition 3,1 Let $\phi, U(\cdot), y$, and $\alpha>0$ be given. Then there exists a unlque temporary equilibrium (TE) corresponding to each $\hat{\theta}>0$ such that

$$
\frac{\alpha}{y^{\prime}(y)} G(\hat{\theta}) \in \partial G(\hat{\theta})
$$

and all TE are of this form.

Proof. We first show that for any TE, $\hat{\theta}$ satisfies (2.22). For any $\theta \in$ $(\hat{\theta}, \hat{\theta}],(3.1 \mathrm{a})$ and $(3.2 a)$ Imply

$$
\theta-\int_{0}^{c(\theta)} S_{\pi}(x) d x \text { and } S_{\pi}(c(\theta))-p(\theta) \text {. }
$$


We have also shown that $p(\theta)>0$ for all $\theta \in \theta$. These facts imply that for all $\hat{\theta} \in[\hat{\hat{\theta}}, \hat{\theta}]$,

$$
c(\theta)=c(\theta)+\int_{\theta}^{\theta} \frac{1}{p\left(\theta^{\prime}\right)} d \theta^{\prime} .
$$

Consider first the case where $s \leq \hat{\theta}$. Then (3.1) and (3.2) imply $p(\theta) c(\theta)-\theta$, and then $(3.11),(3.8)$ and the fact that $\hat{c}-c(\hat{\theta})-y$ imply that:

$$
y=\frac{1}{\lambda} G(\hat{\theta}) .
$$

Consider next the case where $\hat{\theta} \leq \hat{\theta}$. Then (3.6) implies that $p(\underline{\theta})=$ inf supp $\pi$, so that $\pi((p(\theta)))-y$. It follows that $s_{\pi}(c)-p(\theta)$ for all $0<c \leq y$, and hence that

$$
G(\hat{\theta})-\hat{\theta}-F(y)-\int_{0}^{y} S(x) d x-p(\theta) y=\alpha^{-1} y U^{\prime}(y)
$$

Using (3.9), $a^{-1} \mathrm{yU}^{\prime}(\mathrm{y})$, and we obtain (3.12) for this case as wel1.

But condition (3.9) can be rewritten:

$$
\operatorname{Prob}(\theta>\hat{\theta}) \leq \frac{\lambda \alpha}{\mathrm{U}^{\prime}(\mathrm{y})} \leq \operatorname{Prob}(\theta \geq \hat{\theta}),
$$

which is to say

$$
\frac{\lambda \alpha}{U^{\prime}(y)} \in \partial G(\hat{\theta})
$$


The conditions (3.12) and (3.13) then imply that $\hat{\theta}$ satisfies (3.10). For the converse, let $\hat{\theta}$ be any solution to $(3.10)$. We show that exactly one TE can be constructed for this $\hat{\theta}$ value. Let $\lambda^{*}-G(\hat{\theta}) / y$. since $\hat{\theta}>0, \lambda^{*}>0$. Then define $c(\theta)$ by

$$
\begin{array}{ll}
c(\theta)=\frac{1}{\lambda \star} G(\theta) & \text { for all } \theta \leq \theta \leq \hat{\theta}, \\
c(\theta)=y & \text { for all } \theta \geq \hat{\theta} .
\end{array}
$$

Then $c$ is a continuous, non-decreasing, non-negative function, monotonically increasing for all $\theta<\theta<\hat{\theta}$. (If $\hat{\theta} \leq \theta$, these conclusions hold vacuously.)

$$
\begin{aligned}
& \text { Define } p(\theta) \text { by } \\
& \qquad \begin{array}{ll}
p(\theta)-\lambda^{*}[\operatorname{Prob}(\theta \geq \pi)]^{-1} & \text { for all } \theta \leq \pi \leq \hat{\theta}, \\
p(\sigma)=\alpha^{-1} U^{\prime}(y) & \text { for } 811 \quad \gamma>\hat{\theta} .
\end{array}
\end{aligned}
$$

Then $P(\theta)$ is a left-continuous. non-decreasing function, with $p(\theta)>0$ for all $\theta \in \theta$. Clearly the value $\lambda *$ and the functions $c$ and $p$ are uniquely defined.

Since $G$ is monotonically increasing for all $0 \leq \theta \leq \hat{\theta}, G^{-1}$ is well-defined on the interval $\left[0, \lambda^{*} y\right]$ and the functions $c(\theta)$ and $p(\theta)$ just defined imply a unique function $s_{\pi}(c)$ on the interval $(0, y]$ :

$$
S_{\pi}(c)=\lambda^{*}\left[\operatorname{Prob}\left(\theta \geq G^{-1}\left(\lambda^{*} c\right)\right)\right]^{-1}
$$


Let $s_{\pi}(0)=0$ and $s_{\pi}(c)=+\infty$ on $(y, \infty)$. Given $s_{\pi}(c)$, we define a measure $\pi$ on intervals of the form $[0, p]$ and $[0, p)$ by:

$$
\begin{aligned}
& \pi([0, p])-\sup \left(c \geq 0 \mid s_{\pi}(c) \leq p\right) \text { for all } p \geq 0, \\
& \pi([0, p))-\operatorname{lnf}\left(c \geq 0 \mid s_{\pi}(c) \geq p\right) \text { for all } p>0 .
\end{aligned}
$$

This measure $\pi$ can then be uniquely extended to all Borel sets by the operations of set union, intersection, and complementation.

Finally, set $u(\theta)=0$. Then it will be observed from our characterization of consumer and seller optimization that the objects $\left(\pi, S_{\pi}, P, C, U\right)$ so constructed satisfy all the requirements for a TE. $\square$

Proposition 3.2 For any $\alpha>0$, there exists unique TE, corresponding to a unique $\hat{\theta}(\alpha)>0$. Furthermore, $\hat{\theta}(\alpha)$ is a continuous, non-increasing function.

Proof. To prove the existence of a unique $T E$, we need only show the existence of a unique solution to $(3.10)$ for each $\alpha>0$. Since $G(\theta)$ is continuous and strictly increasing in $\theta, \frac{\theta}{y^{\prime}(y)} G(\theta)$ has these same properties as a function of $\theta$. On the other hand, $\partial G(\theta)$ is non-increasing in $\theta$, with a closed graph, and the range $(0, \infty)$. Hence there is a solution $\hat{\theta} \geq 0$ to $(3.10)$ for any $\alpha>0$. Furtherrore, since

$$
\frac{\alpha}{y^{\prime}(y)} G(0)-0<\inf \partial G(0)
$$

and 


$$
\frac{a}{\mathrm{yU}^{\prime}(\mathrm{y})} \mathrm{G}(\tilde{\theta})>0-\sup \partial G(\tilde{\theta})
$$

for all $\bar{\theta}>\bar{\theta}$, all solutions must satisfy $0<\hat{\theta} \leq \bar{\theta}-\sup$ supp $\Phi$. If there are two solutions $\hat{\theta}_{1}<\hat{\theta}_{2} \leq \bar{\theta}$, then one must have $G\left(\hat{\theta}_{1}\right)-G\left(\hat{\theta}_{2}\right)$. which would contradict the fact that $G$ is monotonically increasing for all $\theta \leq \bar{\theta}$.

Thus the function $\hat{\theta}: \mathbf{R}_{++} \rightarrow(0, \bar{\theta}]$ is well-defined, and we consider its behavior as $a$ varies. Let $\alpha_{2}>\alpha_{1}>0$. Then $\hat{\theta}\left(\alpha_{1}\right)$ is the unique $\theta$ for which (3.10) holds, and hence for all $\theta>\hat{\theta}\left(\alpha_{1}\right), \frac{\alpha_{1}}{y^{\prime}(y)} G(\theta)>$ sup $\partial G(\theta)$. Since $a_{2}>a_{1}$, It follows that $\frac{\alpha_{2}}{\mathrm{yU}^{\prime}(\mathrm{y})} \mathrm{G}(\theta)>\sup \partial G(\theta)$ for all $\theta$ $>\hat{\theta}\left(\alpha_{1}\right)$. Then (3.10) cannot be satisfied at $\alpha_{2}$ for any $\theta>\hat{\theta}\left(\alpha_{1}\right)$, which proves that $\hat{\theta}\left(\alpha_{2}\right) \leq \hat{\theta}\left(\alpha_{1}\right)$, or that $\hat{\theta}(\alpha)$ is non-decreasing.

We next show that $\hat{\theta}(\alpha)$ is continuous for all $a>0$. For some sequence $\left(\alpha_{n}\right)$ with $\alpha_{n}>0$ for each $n$ and $\alpha_{n}+a^{*}>0$, suppose that $\theta_{n}-\hat{\theta}\left(a_{n}\right)$ for each $n$, and that $\theta^{\star}-\hat{\theta}\left(a^{*}\right)$. We wish to show that $\theta_{n} \rightarrow$ $\theta^{*}$. That is, for any $\theta^{\prime}$ such that $\theta^{\prime}<\theta^{*}$, we wish to show that $\theta^{\prime}<\theta_{n}$ for all $n$ large enough, and similarly, for any $\theta^{*}$ such that $\theta^{n}>\theta^{*}$, we wish to show that $\theta^{n}>\theta_{n}$ for all $n$ large enough. Consider first the lower bound. Since $\alpha_{n} \rightarrow \alpha$ and $G\left(\theta^{*}\right)>G\left(\theta^{\prime}\right)$, the inequality

$$
\frac{G\left(\theta^{*}\right)}{G\left(\theta^{\prime}\right)} \alpha>a_{n}
$$

must hold for $n$ sufficiently large. We show that this inquality in turn. implies that $\theta_{n}>\theta^{\prime}$. For if one had $\theta_{n} \leq \theta^{\prime}$, one could show that 


$$
\text { inf } \begin{aligned}
\partial G\left(\theta_{n}\right) & \leq \frac{a_{n}}{y U^{\prime}(y)} G\left(\theta_{n}\right) \leq \frac{a_{n}}{y U^{\prime}(y)} G\left(\theta^{\prime}\right) \\
& <\frac{\alpha^{*}}{y U^{\prime}(y)} G\left(\theta^{\prime}\right) \leq \sup \partial G\left(\theta^{*}\right) \leq \inf \partial G\left(\theta_{n}\right),
\end{aligned}
$$

which is a contradiction. The proof for the upper bound $\theta^{\prime \prime}$ is identical.

\section{Stationary Equillbrlum with Independent Monetary Shocks}

We now embed the temporary equilibriun of the previous section in a complete intertemporal equilibrium. We consider an economy operating in an infinity of periods $t=0,1,2, \ldots$ In each period, consumers exchange cash for goods provided by sellers in exactly the manner described in section 2. Cash acquired by sellers is returned to consumers at the end of the period, after the pricing game is completed, in the form of a dividend to shareholders. The monetary authority uses beginning-of-period and end-ofperiod transfers in such a way as to make consumer cash holdings ${ }^{\theta} t$ an independent, identically distributed random variable. In this section we spell out the detalls of this economy, and show that its stationary equilibrium can be obtained by the same construction used to characterize the temporary equilibrium in Section 3.

The economy we consider is made up of a continuum of infinite-lived households, each of which seeks to maximize the expected value of

$$
\sum_{t=0}^{\infty} \beta \tau U\left(c_{t}\right)
$$


where $U$ is the same single-period utility function as above, and $f$ is a discount factor in $(0,1)$. Note that there is no direct utility from cash balances of the kind assumed in (2.10). The monetary authority is assumed to choose a monetary infection $\theta_{t}$ in each period $t$, the value of which is again not known to producers until after all perlod $t$ sales have occurred. The state variable $\left\{\theta_{t}\right\}$ is assumed to be identically and independently distributed across periods, being drawn each period from the same distribution as above. Hence the information of producers at the beginning of period $t$ is simply that $\theta_{t}$ will be drawn from the distribution $\Phi$ on $\theta-[\theta, \bar{\theta}] \subset \mathbf{R}_{++}$as in the temporary equilibrium of the previous section. In this section, we also assume that $\ell<1$.

In order to calculate the endogenously determined value of end-ofperiod cash balances, we must consider how a different level of initial cash balances would affect a given consumer's budget constraint in the following period. Let us recurn again to the finite game discussed at the beginning of Section 2. Let us again assume a continuum of identical players who play identical strategies, except that now a single (non-atomic) consumer i will be assumed to begin the game with money holdings $\mu \theta$ (for some $\mu>0$ ), while all other consumers have $\theta$.

As argued in Section 2, each consumer will bid for as wany units as he can (given the constraint $(2.1)$ ), at each stage prior to the last stage in which he bids for any positive quantity. Furthermore, each of the other consumers bids all of his remaining cash in each of the first n-1 stages. Hence consumer $i$ can bid anything up to $\mu$ times what the others bid, in each of the flrst $n-1$ stages. Thus the most that 1 can purchase in any stage $k<n$ is $\mu c_{k}$ i in stage $n$, consumer 1 can obtain any amount that 
he bids for. The minimum expenditure required for $i$ to purchase $c$ units 1s therefore exactly $\mu$ times the expenditure required for any other consumer to purchase $c / \mu$ units. In the limiting case of a continuum of prices, the required expenditure is thus

$$
\int_{0}^{c / \mu} \min \left[S_{\pi}(x), p(\theta)\right] d x
$$

Note that if the supply function $S_{\pi}$ is defined as in (2.9), for some measure $\pi$, then $\min \left[S_{\pi}(\mathrm{x}), \mathrm{P}\right]$ is an integrable function, and this expression is we11-defined.

Now let $m_{t}^{i}$ denote consumer i's money balances at the beginning of perlod $t$, before the period $t$ monetary injection occurs. The law of motion of this quantity will be

(4.2a) $\quad \mathbf{m}_{t+1}^{i}-\mu_{t}^{i}\left[\theta_{t}-\int_{0}^{c} \frac{i}{t / \mu} \min \left[s_{\pi}(x), p\left(\theta_{t}\right)\right] d x\right]+R_{t}+r_{t}$,

where

$$
\mu_{t}^{i}=\theta_{t}^{-1}\left[\mathbf{m}_{t}^{1}+\theta_{t}-1\right]
$$

Each consumer receives a lump-sum transfer of $\theta_{t}^{-1}$ at the beginning of period $t$, and a lump-sum transfer of ${ }^{{ }} t$ at the end of the period. At the end of the perlod, each consumer also receives as a dividend his proportional share of the total sales revenues $R_{t}$ of the firms. The end. of-period lump-sum transfer is assumed to be 
$(4.2 c)$

$$
{ }_{t}=1-\theta_{t} .
$$

Then, since in a symetric equilibrium the revenues $R_{t}$ equal the expenditure of each consumer, if $m_{t}^{1}-1$ then $m_{t+1}^{i}-1$ as well. We thus consider an equilibrium in which $m_{t}^{1}-1$ forever, in which $\theta_{t}$ again represents the money supply in period $t$, and the expression in (4,2b) indicates the ratio of 1 's post-injection cash balances to those of the typlcal consumer.

The problem of consumer $i$ is then to choose a plan specifying consumption purchases $c_{t}^{i}$ in each period as a function of the history of realizations $\left(\theta_{0}, \ldots, \theta_{t}\right)$ of the monetary infections, so as to maximize the expected value of ( 3.1 ), subject to the constraints that period $t$ expenditure not exceed $\mu_{t}{ }^{\theta} t$, and that $\mu_{t+1}^{i} \geq 0$, in all periods and under all possible histories of monetary injections, given the laws of motion (4.2). Here the determination of $R_{t}$ as a function of the history of monetary injections is also taken as given by the consumer, and similarly the functions $S_{\pi}$ and $P$, which may also depend upon the history of monetary injections. Initial money balances $m_{0}^{1}-1$ for all $i$ are given as an initial condition.

We now specialize to the case of a stationary equilibrtum, in which the functions $\left(p, u, c, s_{\pi}\right)$ are the same for all $t$. As a result one will have $R_{t}-R\left(\theta_{t}\right)$, where the function $R$ is also the same for all $t$. The consumer's problem then takes a stationary zecursive form. Let $v\left(m_{0}^{1}\right)$ denote the maximum attainable value for the expected value of (4.1) given the constraints listed above, for any initial money balances $m_{0}^{1} \geq 1-\ell$. This value function $v$ must satisfy the Bellman equation 


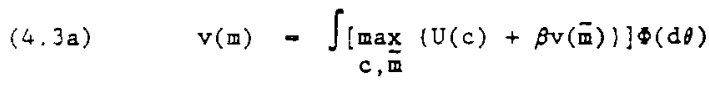

where for each m $\geq 1-\underline{\theta}, \underline{\theta} \leq \theta \leq \vec{\theta},(c, \overline{\mathrm{m}})$ must be chosen such that

$$
\max [1 \cdot \theta, R(\theta)+r(\theta)] \leq \overline{\text { I }} \leq \mathrm{m}+\theta \cdot 1+\mathrm{R}(\theta)+r(\theta),
$$

$$
\tilde{\mathrm{m}}-\mu(\mathrm{m}, \theta)\left(\theta \cdot \int_{0}^{c / \mu(\mathrm{m}, \theta)} \min \left[\mathrm{S}_{\pi}(\mathrm{x}), \mathrm{p}(\theta)\right] \mathrm{dx}\right)+\mathbf{R}(\theta)+r(\theta)
$$

$$
\text { if } \mu(\mathbf{m}, \theta)>0 \text {, }
$$

(4.3d)

$$
c=0 \text { if } \mu(\pi, \theta)=0 \text {. }
$$

Here $\mu(\mathbb{m}, \theta)$ is the function defined in $(4.2 \mathrm{~b}), \quad R(\theta)$ is the function describing aggregate revenues introduced above, and $r(\theta)$ is the function defined in (4.2c). Equation (4.3c) restates the law of motion (4.2a) The lower bound $1-\theta$ in $(4.3 \mathrm{~b})$ follows from the requirement that $\mu_{t+1}^{1} \geq 0$ regardless of the realization of $\theta_{t+1}$. The lower bound $R(\theta)+r(\theta)$ follows from the requirement that the household's expenditure not exceed $\mu_{t}{ }^{\theta} t$, as does (4.3d). Finally, the upper bound in (4.3b) follows from the requirement that $c_{t}^{i} \geq 0$.

This recursive formulation allows us to describe consumer behavior each period by a choice of total purchases $c_{t}^{1}-c\left(m_{t}^{1}, \theta_{t}\right)$, where $(\mathrm{c}(\mathrm{m}, \theta), \bar{m}(\mathrm{~m}, \theta))$ is the solution to the maximization problem inside the brackets in (4.3a). This will in turn allow us to describe a stationary intertemporal equilibrium as a succession of temporary equilibria of the 
kind analyzed in the previous section. But since a statlonary equilibrium involves the value function $v(m)$, before we can define a stationary equilibrium we need to ensure the existence of a solution to (4.3) (Proposition 4.1) and to establish its differentiablity at m - 1 (Proposition 4.2). These results develop some other properties of $v$ and the associated policy functions $(c, \tilde{m})$ as well.

Proposition 4.1. In the maximization problem (4.3), suppose that

(i) the function $p(\theta)$ is bounded and left-continuous, with $p(\theta)>$ 0 for all $\theta \in \theta$;

(ii) the function $S_{*}$ (c) is non-decreasing and left-continuous, with $S_{\pi}(c) \geq p(\theta)$ for all $c \geq 0$, and with $S_{\pi}(c)=+\infty$ for all $c>$ $\mathrm{y}$;

(iii) the function $R(\theta)$ is continuous, with $R(\theta) \geq 0$ for all $\theta \in$ $\theta$; and

(iv) the function $U(c)$ is bounded, continuous, strictly increasing, and concave, for all $c \geq 0$.

Then there exists a value function $v(m)$, defined for all $m \geq 1-\underline{\theta}$, that satisfies the Bellman equation (4.3a). Furthermore, $v(m)$ is bounded, continuous, strictly increasing, and concave.

Proof: See the appendix.

By a standard argument (see, e.g., Stokey and Lucas, Theorem 9.2), the existence of a function $v(\mathbb{m})$ that satisfies the Bellman equation Implies the existence of a solution to the original infinite-horizon consumer optimization problem, and optimal behavior consists of choosing each period 


$$
\begin{aligned}
& c_{t}^{i}=c\left(m_{t}^{1}, \theta_{t}\right), \\
& m_{t+1}^{i}=\tilde{m}\left(m_{t}^{i}, \theta_{t}\right),
\end{aligned}
$$

where $c(m, \theta), \tilde{m}(m, \theta)$ are the functions that solve the single period optimization problem in (4.3a). We accordingly turn our attention to that single period problem.

In a stationary equilibrium, we wish to specify $R(\theta)$ as

$$
R(\theta)-\int_{0}^{c(\theta)} \min \left[S_{\pi}(x), p(\theta)\right] d x
$$

where $c(\theta)=c(1, \theta)$. (Note that we have not yet proved that this is possible, since we have only shown the existence of a function $c(\theta)$ given some specification of $R(\theta)$--we do not know that we can find a function $R(\theta)$ such that the $c(\theta)$ that satisfies (4.3a) will also generate that same function $R(\theta)$ in (4.4).) Then given the initial condition $m_{0}^{i}-1$ for all $i$, each household's optimal behavior will be to choose

$$
\begin{aligned}
& c_{t}^{1}-c\left(\theta_{t}\right), \\
& m_{t+1}^{i}=1,
\end{aligned}
$$

in all periods. (This follows from $(4.3 \mathrm{c})$.)

Hence it suffices to consider the single period maximization problem in (4.3a) for the case m $=1$. Suppose that, as assumed in the prevtous section, $U(c)$ is continuously differentiable for all $c>0$, strictly 
concave, and that $\lim _{c \rightarrow 0} U^{\prime}(c)-+\infty$. Suppose also that $v(m)$ is

differentiable at $m-1$. Then necessary and sufficient conditions for the function $c(\theta)$ to solve the single period maximization problem (given that $R(\theta)$ satisfies $(4.4)$ ) are

$$
U^{\prime}(c(\theta)) \geq \beta V^{\prime}(1) \min \left[S_{\pi}(c(\theta)), p(\theta)\right]
$$

$$
\int_{0}^{c(\theta)} \ln \left[S_{\pi}(x), p(\theta)\right] d x \leq 0
$$

for all $b \in \theta$, and that for each $\theta$, elther (4.5a) or (4.5b) holds with equality. But these are Just the Kuhn-Tucker conditions for the maximization of (2.10a) subject to (2.10b) if the parameter a in (2.10a) is given the value

$$
\alpha-\beta v^{\prime}(1) \text {. }
$$

Thus with this identification of $\alpha$, consumer behavior each period in a stationary equilibrium is exactly of the kind described in the previous section.

Sufficlent conditions for the differentiability of the value function. and hence for the characterization (4.5) of consumer behavior, are given by the following result.

Proposition 4.2. In addition to the hypotheses of Proposition 4.1, suppose that 
(i) the function $U(c)$ is continuously differentiable for all $c>$ 0 , it is strictly concave (i.e., $U^{\prime}(c)$ is monotonically decreasing), and $\lim _{c \rightarrow \infty} U^{\prime}(c)=+\infty$;

(11) $\mathrm{S}_{\pi}$ (c) $<+\infty$ for some $c>0$; and

(iii) the function $c(\theta)$ that solves the maximization problem in (4.3c) for the case m - 1 is such that (4.4) holds, and furthermore such that

$(4.7)$

$$
s_{\pi}(c(\theta)) \leq p(\theta)
$$

for all $\theta \in \theta$.

Then the value function $v(m)$ that satisfies (4.3a) is differentiable at $m=1$, and the derivative equals

$$
V^{\prime}(1)-(1-\beta)^{-1} \int_{\theta}^{-1} U^{\prime}(c(\theta)) c(\theta) \Phi(d \theta)
$$

Proof. See the appendix.

Hence the characterization of optimal consumer behavior in the previous section continues to apply to a stationary equilibrium, with the identification of the parameter $\alpha>0$ given by (4.6) and (4.8). We next consider optimal producer behavior. Each producer $f$ has a capacity constraint $y>0$ in each period. The producer chooses a supply measure $\pi_{t}^{j}$ in period $t$ that satisfies (2.2). This supply measure results in revenues $R_{t}^{j}\left(\theta_{t}\right)$ as a function of the realized monetary injection $\theta_{t}$ ' given by $(2.8)$. 
Producers distribute these earnings to the households (owners of the firms) at the end of the period, and producer $J$ chooses $\pi_{t}^{J}$ so as to maximize the value to the representative household of an increment in 1 ts earnings distribution, contingent upon $\theta_{t}$, of the form $R_{t}^{J}\left(\theta_{t}\right)$. The value to be maximized is an ex ance value, before the value of ${ }^{\theta_{t}}$ is known, and firm $J$ takes as given the aggregate earnings distribution function $R\left(\theta_{t}\right)$ In calculating the value of an incremental distribution. Then, under the hypotheses of Proposition 4.2, $\pi_{t}^{J}$ is chosen so as to maximize

$$
\int_{R_{t}^{j}}^{j}(\theta) v^{\prime}\left(\tilde{m}\left(m_{t}, \theta\right)\right) \Phi(d \theta)-v^{\prime}(1) \int R_{t}^{j}(\theta) \Phi(d \theta)
$$

That is, it is chosen so as to maximize expected revenues, given that ${ }^{\theta} t$ will be drawn from the distribution $\$$. Thus $\pi$ is chosen each perlod in exactly the way assumed in the temporary equilibrius of the previous section, and the characterization of optimal producer behavior there applies here as well.

Thus under the hypotheses of Proposition 4.2, the characterizations of both producer and consumer behavior in the previous section continue to apply. As a result, a stationary equilibrium will involve a succession of temporary equilibria of the kind described earlier. But our previous characterization of temporary equilibrium implies that the hypotheses of Proposition 4.2 will indeed hold in such a case. Hence we may define a stationary equilibrium as follows: 
Definition. A stationary equilibrium is a constant $\alpha>0$, a measure $\pi \in$ $M$, a function $S_{\pi}:[0, y] \rightarrow \overline{\mathbf{B}}_{+}$defined in terms of $\pi$ by (2.9), and a triple of measurable functions $p: \theta \rightarrow \overline{\mathbf{R}}_{+}, u: \theta \rightarrow[0,1]$, and $c: \theta \rightarrow \overline{\mathbf{R}}_{+}$, such that

(i) given $\alpha>0,\left(\pi . S_{\pi}, p, u, c\right)$ constitute a temporary equilibrium in the sense defined in Section 2; and

(ii) the constant a satisfies

$$
\alpha-\beta(1-\beta)^{-1} \int_{\theta}^{-1} U^{\prime}(c(\theta)) c(\theta) \Phi(\mathrm{d} \theta)
$$

Here (4.9) follows from (4.6) and (4.8).

The following result shows that such an equilibrium exists for all possible distributions of the monetary shocks.

Proposition 4.3. Suppose that the function $U(c)$ is bounded, continuous, strictly increasing, and strictly concave, for all $c \geq 0$, continuously differentiable for all $c>0$, and satisfies $\underset{c \rightarrow 0}{\lim } U^{\prime}(c)-+\infty$. Then there exists a stationary equilibrium.

Proof. By Proposition 3.2, there is a unique TE for every $\alpha>0$, and $\hat{\theta}(\alpha)$, the unique solution to (3.10), is a continuous, non-increasing function of $\alpha$, with $\hat{\theta}: \mathbf{R}_{+} \rightarrow(0, \bar{\theta}]$.

We next consider how the function $c(\theta ; \alpha)$ in a TE with given $a$ varies with $\alpha$. Ror any $\alpha>0$, the construction used in the proof of Proposition 3.1 implies that 


$$
c(\theta ; \alpha)=y \min \left[\frac{G(\theta)}{G(\hat{\theta}(\alpha))}, 1\right] .
$$

Now for pairs $(\theta, \alpha)$ such that $\theta \in \theta, \alpha>0$, and $\hat{\theta}(\alpha) \geq \theta$, the continulty of $G(\theta)$, the continuity of $\hat{\theta}(\alpha)$, and the fact that $G(\hat{\theta}(\alpha)) \geq G(\theta) \geq G(\theta)$ $>0$ imply that $G(\theta) / G(\hat{\theta}(\alpha))$ is a continuous function of $(\theta, \alpha)$. Moreover, this function equals 1 at all points on the boundary where $\hat{\theta}(\alpha)$

- $\theta$. Hence $c(\theta ; \alpha)$ is a continuous function of $(\theta, \alpha)$ on the domain $\theta \times R_{++}$. Furthermore, if one defines

$$
c(\theta ; 0)-y G(\theta) / G(\theta)
$$

then $c(\theta ; \alpha)$ is a continuous function on the dowain $\theta \times \mathbf{R}_{+}$.

The function $c(\theta ; \alpha)$ is obviously non-decreasing in both arguments on that domain. Furthermore, one observes that for any $\theta \in \theta, \lim _{\alpha \rightarrow \infty} c(\theta ; \alpha)-y$. Finally, the function is bounded and bounded away from zero on $\theta \times \mathbb{R}_{+}$, insofar as

$$
0<y G(\theta) / G(\bar{\theta}) \leq c(\theta ; \alpha) \leq y
$$

for all $\theta \leq \theta \leq \bar{\theta}, \alpha \geq 0$.

Now consider the right hand side of $(4,9)$, as a function of $\alpha$.

Because $U(c)$ is continuously differentiable for all $c>0$, and $c(\theta ; \alpha)$ is continuous in both arguments, bounded, and bounded away from zero, the function

$\frac{c(\theta ; \alpha) U^{\prime}(c(\theta ; \alpha))}{\theta}$ 
is a continuous function of $(\theta, \alpha)$ on the domain $\theta \times \mathbb{R}_{+}$that is both bounded and bounded away from zero. Then by the Lebesgue dominated convergence theorem, the integral of this function over $\theta$ is a continuous function of $\alpha$ on the domain $\alpha \geq 0$, and is both bounded and bounded away from zero. Hence the right hand side of (4.9) is a function of $\alpha$ with these properties.

It follows that both the left hand and right hand sides of (4.9) are continuous functions of $\alpha$, with the left hand side necessarily larger for large enough $\alpha$, and smaller for small enough $a>0$. Hence there must exist a solution for some $\alpha>0$. Given this value, the $\left(\pi, s_{\pi}, p, u, c\right)$ that describe the temporary equilibrium for this value of $\alpha$ then constitute a stationary equilibrium.

\section{Discussion}

We now consider some properties of the stationary monetary equilibrium just shown to exist. The stationary equilfbrium consists of a sequence of temporary equilibria of the kind characterized in section 3 . In each period $t$ there is another independent drawing of the shock ${ }^{\theta} t$ tha: determines the period's money supply. The aggregate purchases that result are given by $c_{t}-c\left(\theta_{t}\right)$, and the marginal price level (the highest price $a$ : which all goods offered for sale are sold) is given by $p_{t}=p\left(\theta_{t}\right)$. The distribution of transaction prices in period $t$ is given by the measure * truncated at the price $P_{t}$, and total nominal spending is given by $R_{t}$ $R\left(\theta_{t}\right)=\min \left(\theta_{t}, \hat{\theta}\right)$. The $i . i . d$. random variations $\operatorname{la} \theta_{t}$ thus give rise to i.i.d. variations in spending, consumption, and prices. 
Under the interpretation in which producers begin with a productive capacity rather than an endowment of goods and produce only to f1ll the orders that they accept, the fluctuations in $c_{t}$ represent fluctuations in real output. As promised, then, we have exhlbited a model in which surprise variations in the money supply affect not only nominal spending and prices, but real activity as well. Because $c(\theta)$ is a non-decreasing function, monotonically increasing over the range $\theta \leq \theta \leq \hat{\theta}$, low realizations of the money supply are assoclated with low levels of output. Thus the model can rationalize the association between low rates of growth of the money supply and contractions of economic activity documented by authors such as Friedman and Schwartz (1963). Indeed, a lower money supply necessarily means lower output in this equilibrium, except if one is comparing values of $\theta$ in the range $(\theta>\hat{\theta})$ for which nominal spending is unaffected by changes in the money supply. ${ }^{5}$ The marginal price level is also non-decreasing in $\theta$, so that higher reallzations of the money supply are generally associated with higher prices being reached. The average transaction price $\bar{p}_{t}$ is given by $\tilde{p}_{t}=\tilde{p}\left(c_{t}\right)$, where

$$
\tilde{p}(c)=\frac{1}{c} \int_{0}^{c} s_{\pi}(x) d x .
$$

Because the supply curve $S_{\pi}(c)$ is non-decreasing, with $S_{\pi}$ (c) $>S_{\pi}(c(\underline{c}))$ for all $c>c(\theta)$, the function $\tilde{p}(c)$ is monotonically increasing. Thus the model predicts an upward-sloping Ph1llips curve relation linking deviations of output from its average level (or from capacity) to corresponding deviations of average transactions prices from the level that would have been expected prior to realization of the monetary shock. 
In this model, as in that of Lucas (1972), variations in the money supply affect real activity only because they are unanticlpated. Consider, by way of contrast, the consequences if the drawings of ${ }^{\theta} t$ were made public before any trading occurred. Again a stationary equilibrium would exist. Again $\alpha$ would be independent of the realization of ${ }^{\theta}{ }_{t}$, and consumers would purchase goods until the marginal price of goods reached the value $a^{-1} U^{\prime}(c)$. Producers would be able, however, to choose a supply measure $\pi_{t}$ contingent upon $\theta_{t}$, and since there would be no uncertainty about the marginal price $P_{t}$, each producer would offer to sell $y$ units at the price $P_{t}$, and none at any lower price. Furthermore, in equilibrium no producers would be rationed; hence one would have $c(\theta)-y$ for all $\theta$. It follows that the equilibrium price would be given by

$$
p(\theta)=\min \left[\frac{\theta}{y}, \frac{U^{\prime}(y)}{\alpha}\right] .
$$

Thus for all $\theta \leq \theta \leq \hat{\theta}$ (i.e., in the range over which changes in the money supply affect nominal spending), prices increase proportionally with $\theta$. The resulting equilibrium would in fact be identical to the equilibrium of a cash-in-advance model with Walrasian spot markets (see, e.g., Sargent, 1987, chap. 5). 6

It is also worth noting that in this model, uncertainty about the money supply results not only in more variable real activity, but in a lower average level of output as well. As we have fust argued, if there were no monetary uncertainty, output would equal capacity in all periods. In the equilibrium with unanticipated variations in $\theta_{t}$, output depends upon the realization of $\theta_{t}$, but it never exceeds capacity. Hence on average it is below capacity, and so below its average value in the absence of such 
uncertainty. ${ }^{7}$ Th1s is an 1mportant difference between the present model and models such as that of Lucas (1972), or the models in which producers are commited to sell any amount demanded at a nominal price that is fixed in advance. In those models, an unexpectedly hish money supply results in output greater than the equilibrium level of output in the sbsence of monetary uncertainty, symmetrically with the output reduction induced by a low money supply, and monetary uncertainty reduces welfare mainly by increasing output yariability.

In the equilibrium displayed above, monetary shocks produce variations in output that are unrelated to any change in inputs; hence they produce variations in productivity as conventionally measured, even though the production technology is not actually changing. Thus imagine a variation on our model in which capacity is proportional to the firm's number of employees, each of whom works a fixed shift. Assume that each period's employment is chosen before orders begin to be taken, that labor contracting occurs before the realization of $\theta_{t}$, and that households supply labor inelastically. Whether or not we assume that the wages contracted for can be made contingent upon the reslization of ${ }^{t}$, each firm's wage bill will be independent of its sales, and so the firm will price its output so as to maximize expected revenues as assumed above. The equilibrium will be the same as above, except that now there will exist no pure profits on average. Since the quantity of labor hired will not vary with ' ${ }_{t}$ ' the Solow residual will equal the percentage change in sales. Thus monetary surprises will produce variation in the Solow residual, correlated with output variation. One could also extend the model to allow for variable inputs in addition to the expenditure on capacity. In this case, inputs would vary with ${ }_{t}$ but the Solow residual would still move procyclically, because payments to the 
variable factors would on average be less than total revenues, due to the positive price of capacity. (This kind of effect is discussed in detail by Eden (1990a) and Rotemberg and Sumers (1990).)

This result contrasts with models in which production is always efficient and factors are competitively priced, and also with models in which prices are fixed in advance, if the price is fixed at a level that makes price equal expected marginal cost (as, for example, in King (1991)). If instead prices are set to exceed marginal cost on average because firms have market power (as in Svensson (1986) and Blanchard and Klyotaki (1987)), the variations in output resulting from monetary shocks will cause procyclical movements in the Solow residual, for the reason stressed by Hall $(1988,1990) .^{8}$ The result occurs in the present model for somewhat the same reason--price exceeds marginal cost in all states in which the capacity constraint does not bind, so that the share of variable input costs in total revenues is an underestimate of the true elasticity of output with respect to variable input quantity.-but here this outcome does not require market power.

In the present model, as in that of Lucas (1972), producers' imperfect information about the state of nominal aggregate demand is crucial to the non-neutrality of monetary shocks. But both the precise character of the information that producers lack and the mechanisu through which this ignorance affects equilibrium activity are quite different in the two models. In Lucas (1972), the real effects of monetary shocks depend upon the existence of informationally isolated markets (islands). Sellers in each market are aware of the price and volume of trade in that market, but not of prices in the economy as a whole. In the present model, the real effects of money do not depend upon informational fsolation of this kind.. 
as was noted in Section 2, we may assume if we like that all sales are public, economy-wide.

The models also differ in the way that imperfect information affects equilibrium output. In both models, a recession occurs in the case of an unexpectedly low money supply because producers refuse to sell their output at prices low enough to allow consumers to buy as much as they would in a full-information equilibrium. But the reason that producers restrict supply (compared to what they would do had they better information) is different in the two cases. In the model of Lucas (1972), there is no misperception as to the price at which any producer would be able to sell his goods, because each producer knows the market-clearing price before accepting any orders. What producers are mistaken about, instead, is the shadow value to them of the cash that they would recelve from additional sales. Sellers supply too little at the equilibrium price because they fall to realize how low prices generally will be in the following period, when they spend the cash obcained from current sales, and thus how wllling they should be in the current perlod to supply goods at a low money price. They would know this if they knew the current money supply shock, because the reduction in the woney supply is permanent, and prices fall proportionally after a one-period delay. In the present model, by contrast, producers correctly understand the shadow value of cash ( $\beta v^{\prime}(1)$ in our notation above); knowledge of the current shock $\theta_{t}$ would not affect their evaluation of this value. The information that they lack, instead, is about how the price they charge $\forall 1 .:$ affect the quantity that they will be able to sell. In a recession stace producers offer to supply too few goods at low money prices, because they overestimate the chance of eventually finding buyers who will pay a high 
price; if they knew the current state of nominal aggregate demand they would know betrer.

Our observation that knowledge of the current money supply would not affect producers' estimate of the shadow value of additional cash in the present model follows from our unrealistic assumption that the money supply is independently distributed across periods. If we were to assume instead that a higher realization of ${ }^{\theta} t$ implied higher money supplies, on average, in all subsequent periods as well, then one's estimate of ${ }_{t}$ would affect one's estimate of $\beta V^{\prime}(1)$, and misperception of the shadow value of cash due to ignorance of the current money supply would indeed bear some of the blame for the inefficient use of resources associated with monetary instability. But a still more realistic model would recognize that each producers' sales are stochastic for many reasons independent of the current realization of money growth, and as a result that there is little reason for producers to revise their estimates of either the current or the future money supply on the basis of surprise variations in their own current sales. Consider, for example, a model with many submarkets each organized along the non-Walrasian lines explained in Section 2, but with independent variations in spending in the various submarkets superimposed upon the (possibly autocorrelated) variation in aggregate spending. We would expect each submarket's equilibrium to be similar to the kind described above, with producers choosing a pricing strategy similar to the one that would maximize expected revenues, even in the case of significant persistence in the fluctuations in aggregate spending.

There is an alternative interpretation of the equilibrium of section 4, under which consumers--symetrically with firms-learn the current realization $\theta_{t}$ only in the course of trading. Instead of assuming that $\theta_{t}$ 
is revealed to consumers at the beginning of the period, when they receive a single transfer that brings their cash balances to the level $\theta_{t}$ ' suppose that consumer cash balances are $\ell$ at the beginning of trading in all periods, and that additional transfers occur continuously during trading, stopping when a total money supply of ${ }_{t}$ per household has been reached. The drawing of $\theta_{t}$ by the monetary authority is thus not revealed to anyone--consumers or firms--until the transfers stop. At each stage in this process, consumers' knowledge of $\theta_{t}$ is limited to knowledge of the distribution $\Phi$ and of the fact that $\theta_{t}$ is at least as large as the amount of cash already received in the current period. If in equilibrium consumers spend all of the cash avallable to them as soon as they receive it, up to the point where $\alpha^{-1} U^{\prime}(c)$ no longer exceeds the marginal price at which goods are available, then the information of producers and consumers will be the same at all stages: Each will know only the ex ante distribution of possible money supplies, and the quantity of money transferred and spent up to that point.

In such a symmetric-information variant of the model, equilibrium transactions are the same as before. The only difference is that now consumers have less cash with which to bid in the early stages of the trading game, and so make bids that are proportionally smaller, to obtain the same quantity of goods at the same prices as before. The equivalence of the two models is most easily seen if one considers the case of a distribution $\Phi$ with a finite support, $\left(\theta_{0}, \theta_{1}, \ldots, \theta_{N}\right)$, with $0<\theta_{0}<\theta_{1}<$ $\ldots<\theta_{N}$. Suppose that consumers begin trading with cash balances of $\theta_{0}$. that subsequently an additional transfer of $\theta_{1}^{-\theta_{0}}$ is made with probability $\pi_{1}$, then a transfer of $\theta_{2}^{-\theta}$. with probability $\pi_{2} / \pi_{1}$ (conditional upon the first transfer having been made), and so on, until finally a transfer of 
$\theta_{N}^{-\theta} N-1$ is made with probability $\pi_{N} / \pi_{N-1}$ (conditional upon the $N-1$ previous transfers having been made), where $0<\pi_{N}<\pi_{N-1}<\ldots<\pi_{1}<1$. Suppose furthermore that there is time between transfers for a complete bargaining process of the kind described in Section 2 to occur; in each such stage producers can offer to supply only that part of their capacity not already committed, and consumers can spend only that cash already received and not already spent.

It is obvious from our previous analysis that in each stage, all goods sold will be sold at the same price ( $p_{k}$ when the money supply is $\theta_{k}$, for $k$ $=0,1, \ldots, N)$. It is also obvious that all cash available will be spent in each stage $k$ prior to the last stage in which any goods are sold (call it stage $n$ ); that the prices in the several stages will satisfy $p_{k} \pi_{k}-p_{0}$ for each $1 \leq k \leq n$; that the total quantity sold in the first $n$ stages will equal $y$; and that $a^{-1} U^{\prime}(y)$ exceeds $\pi_{k}^{-1} p_{0}$ for each $k<n$, is no smaller for $k-n$ (with equality if there is unspent cash in stage $\pi$ ), and is no larger for $k>n$. Thus the equilibrium prices and quantities are the same as if the the value of $\theta_{t}$ were revealed to consumers immediately, and a single trading game of the kind described in Section 2 were played.

In this case of the sequence of symmetric-information trading games, each stage's trading is equivalent to a Walrasian equilibrium. Thus the symmetric-information model just described is equivalent to that of Eden (1990b, 1991), in which there is a sequence of Walrasian spot markets, one after each new injection of cash. ${ }^{9}$ There is thus a sense in which our results do not rely upon a non-Walrasian market structure, if the sequential revelation of information about the state of nominal aggregate demand can be motivated in some other way. We are attracted to the non-Walrasian structure described in Section 2 because it seems to us to capture some 
important elements of economic reality, but it is not required to deliver the effects we derive from it. 


\section{Appendix: Proofs of Propositions 4,1 and 4.2.}

For convenience, we restate the propositions in the text.

Proposition 4.1. In the maximization problem (4.3), suppose that

(1) the function $p(\theta)$ is bounded and left-continuous, with $p(\theta)>$ 0 for all $\theta \in \theta$;

(11) the function $S_{\pi}(c)$ is non-decreasing and left-continuous, with $s_{\pi}(c) \geq p(t)$ for all $c \geq 0$, and with $s_{\pi}(c)-+\infty$ for all $c>$ $y$;

(iii) the function $R(\theta)$ is continuous, with $R(\theta) \geq 0$ for all $\theta \in$ $\theta$; and

(1v) the function $U(c)$ is bounded, continuous, strictly increasing, and concave, for all $c \geq 0$.

Then there exists a value function $v(m)$, defined for all m $\geq 1-\theta$, that satisfies the Bellman equation (4.3a). Furthermore, $v(m)$ is bounded, continuous, strictly increasing, and concave.

Proof: The proof involves five parts. We first, (1), use the constraincs $(4.3 b)-(4.3 d)$ to express the decision variable $c$ in (4.3a) in terms of Then, (2), we define an operator $T$ associated with (4.3) and show that this operator takes the set of bounded continuous functions on $(1-\theta, \infty)$ lnto itself. We show, (3), that $T$ has a unique fixed point $v$ in this set of functions, the unique solution to (4.3). Then we show, (4), that $v$ is increasing and, (5) that $\mathrm{v}$ is concave.

(1) For each $\mathbf{m} \geq 1-\theta, \theta \in \theta$, let $\Gamma(m, \theta)$ denote the interval of values for i that satisfy $(4.3 b)$. Note that $\Gamma$ is a continuous, compac: valued correspondence. Let $D$ denote the graph of $\Gamma$, 1.e., the set 
$(m, \theta, \overline{\mathrm{m}})$ satisfying the inequalities just mentioned. For any $\theta \in[(\hat{\theta}, \bar{\theta}]$, let $D_{\theta}$ denote the set of (m, $\left.\bar{m}\right)$ such that $(\pi, \theta, \tilde{m}) \in D$, and for any subinterval $N \subseteq[\theta, \bar{\theta}]$, let $D_{N}$ denote the subset of $D$ for which $\theta \in N$ Note that for any $\theta \in[\beta, \bar{\theta}], D_{\theta}$ is a closed, convex set.

Now for any $(m, \theta, \bar{m}) \in D$, there exists a unique $c \geq 0$ satisfying $(4.3 c)-(4.3 d)$. For all $(m, \theta)$ such that $\mu(m, \theta)>0$, the right hand side of (4.3c) is monotonically decreasing in $c$. When $c-0$, it equals the upper bound in ( $4.3 \mathrm{~b})$, while as $c$ is made arbitrarily large, it becomes an arbitrarily large negative quantity. Thus for any value of $\bar{m}$ within the bounds (4.3b), there exists a unique solution $c \geq 0$. Let this be denoted $c(m, \theta, \bar{m})$. We wish to consider further the continuity of this function.

For any $\bar{\theta}>\ell$, there exists a left nelghborhood $N$ of $\bar{\theta}$ on which $P(\theta)$ is continuous. Let $\mathrm{N}$ be chosen so that $\theta$ is bounded away from $\underline{\theta}$. Then $\mu(m, \theta)$ is bounded away from zero on $D_{N}$, and hence

$$
\mathrm{J}(\mathbf{m}, \theta, \tilde{\mathbf{m}})=\theta-\frac{\overline{\mathbf{m}} \cdot \mathrm{R}(\theta) \cdot \tau(\theta)}{\mu(\mathbf{m}, \theta)}
$$

is a continuous function on $D_{N}$. Furthermore,

$$
I(z, \theta)=\int_{0}^{z} \min \left[S_{\pi}(x), p(\theta)\right] d x
$$

is a continuous function for all $\theta \in N, z \geq 0$, and monotonically increasing in $z$. Hence the equation

$$
I(\mathbf{z}, \theta)-J(\mathbf{m}, \theta, \overrightarrow{\mathbf{m}})
$$


has a unique solution $z(m, \theta, \overline{\mathrm{w}})$, and this latter function is continuous on $D_{\mathrm{N}}$. Then the unique solution to $(4.3 \mathrm{c})$ is given by

$$
c(\mathbb{m}, \theta, \tilde{\mathbf{m}})-\mu(\mathbf{m}, \theta) z(\mathbf{m}, \theta, \tilde{\mathbf{m}})
$$

and this is continuous on $D_{N}$ as well. (Note that the above functions are all well-defined at all points in $D$ where $\mu(m, \theta)>0$, but $I(z, \theta)$, $z(m, \theta, \overline{\mathrm{m}})$, and $c(\mathbb{m}, \theta, \overline{\mathrm{m}})$ need not be continuous on this entire domain.)

Let us next consider the case $\theta-\theta$. In this case, $I(z, \theta)-p(\theta) z$. Hence for all m $>1-\theta$ (so that $\mu(\mathbf{m}, \theta)>0$ ), the unique solution to (A.I) is

$$
z(\mathrm{~m}, \theta, \overline{\mathrm{m}})-\frac{\hat{\theta}[\mathrm{m}-\overline{\mathrm{m}}+\mathrm{R}(\hat{\theta})]}{\mathrm{p}(\hat{\theta})[\mathrm{m}+\hat{\theta} \cdot 1]}
$$

which implies

$$
c(\mathrm{~m}, \theta, \tilde{\mathrm{D}})-\frac{\mathrm{m}-\tilde{\mathrm{m}}+\mathrm{R}(\boldsymbol{\theta})}{\mathrm{p}(\boldsymbol{\theta})} .
$$

On the other hand, if $\mathrm{m}-1-\phi,(m, \phi, \overline{\mathrm{m}}) \in \mathrm{D}$ requires that $\overline{\mathrm{m}}-$ $R(A)+1-\theta$, so that the numerator of (A.2) is zero. In this case, (4.3d) requires that $c-0$, so (A.2) applies in this case as well. From (A.2) it is evident that $c(m, \phi, \bar{m})$ is a continuous function of $(m, \tilde{m})$ on $D_{\ell}$.

(2) Let $f(\mathbb{m})$ be any bounded, continuous function, defined for all $\mathrm{m}$ $\geq 1-\theta$. Then

$$
\mathrm{F}(\mathbf{m}, \theta, \overline{\mathbf{m}})=\mathrm{U}(\mathbf{c}(\mathbf{m}, \theta, \overline{\mathbf{m}}))+\beta \mathbf{f}(\overline{\mathbf{m}})
$$


is a bounded function on D. Furthermore, it follows from our results above that for any $\bar{\theta}>\theta$, there exists a left neighborhood $N$ of $\bar{\theta}$ such that $F$ is continuous on $D_{N}$, and that $F(m, \theta, \bar{m})$ is a continuous function of (m, 畐) on $\mathrm{D}_{\underline{\theta}}$.

Now for any m $\geq 1-\theta, \theta \leq \theta \leq \bar{\theta}$, define

$$
\phi(\mathrm{m}, \theta)-\sup _{\tilde{\mathrm{m}} \in \Gamma(\mathrm{m}, \theta)} F(\mathbf{m}, \theta, \tilde{\mathrm{m}}) .
$$

For any $\vec{\theta}>\theta$, let $N$ be the left neighborhood of $\tilde{\theta}$ just referred to. Then by the theorem of the maximum (Stokey, Lucas, and Prescott, Theorem $3.6), \phi(m, \theta)$ is continuous on the set $m \geq 1-\theta, \theta \in \mathbb{N}$. Furthermore, for $\theta$ - $\theta, \phi(m, \theta)$ is a continuous function of $m$ on the set $m \geq 1-\theta$. Thus we observe that $\phi(m, \theta)$ is a well-defined bounded function for all m $\geq 1-\theta$, $\theta$ $\leq \theta \leq \bar{\theta}$; that for every $\theta \in \theta, \phi(m, \theta)$ is a continuous function of $m$; and that for every $m \geq 1-\theta, \phi(m, \theta)$ is a left-continuous function of $\theta$. Finally for any $m \geq 1-\underline{\phi}$, define

$$
(T f)(m)=\int_{\phi(m, \theta) \Phi(d \theta)} .
$$

Since $\phi(m, \theta)$ is a bounded, left-continuous function of $\theta$, it is integrable, and the above expression is well-defined. It is also obviously bounded as a function of $m$. We wish to show that it is also continuous. Consider any sequence $\left(m_{n}\right)$ such that $m_{n} \geq 1-\theta$ for each $n$, and $m_{n}+m$. Since $\phi(m, \theta)$ is continuous in $m$ for every $\theta$, the functions $\left(\phi\left(m_{n^{\prime}}\right)\right)$ converge pointwise to the function $\phi(m,)^{*}$. All of those functions are 
integrable, and they are uniformly bounded; hence, by the Lebesgue dominated convergence theorem,

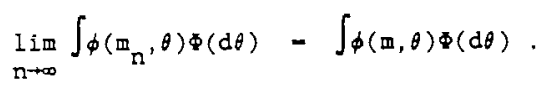

Thus $\mathrm{Tf}$ is a continuous function of $\mathrm{m}$.

We observe that (A. 3 ) defines on operator $T$, mapping the set of bounded continuous functions on $\left(1-\theta_{1}+\infty\right)$ into itself. The existence of a function $v(m)$ satisfying (4.3a) then follows if we can show that there exists a fixed point of the mapping $T$.

(3) Let $\mathbf{F}$ denote the set of bounded continuous functions on $[1-\ell,+\infty)$, with the sup norm. For any functions $f, g, \in \mathbf{P}, f \geq g$ implies that $\mathrm{Tf} \geq \mathrm{T}_{\mathrm{g}}$. For any function $f \in \mathbf{P}$ and any constant function $a$, $T(f+a) \leq T F+\beta a$. Thus the Blackwell conditions are satisfied (recall that $0<\beta<1$ ), and so $T$ is a contraction (Stokey, Lucas, and Prescott, Theorem 3.3). Then since $F$ is a complete metric space, $T$ has a unique fixed point $v \in P$. This function $v$ necessarily satisfies (4.3a).

(4) We next show that $v(m)$ is monotonically increasing. Let $F^{\prime} \subset F$ be the set of bounded, continuous functions that are also non-decreasing. We wish to show that $T$ maps $\mathbf{P}^{\prime}$ into itself as well.

Note that $\Gamma$ is increasing in $m$, in the sense that $m^{\prime} \geq m$ implies that $\Gamma(m, \theta) \subseteq \Gamma\left(m^{\prime}, \theta\right)$. Note also that $\mu(m, \theta)$ is strictly increasing as a function of $m$, as a result of which $\mathrm{J}(\mathrm{m}, \theta, \overline{\mathrm{m}})$ is strictly increasing in m on the subset of $D$ where $\mu(m, \theta)>0$ (so that $J$ is defined). Since $I(z, \theta)$ is strictly increasing in $z$, equation (A.1) defines a function $z(m, \theta, \tilde{m})$ that is strictly increasing in $m$ (on the domain of $\mathrm{J}$ ). It 
follows that $c(m, \theta, \tilde{m})$ is also strictly increasing in $m$ on this domain. Consider now the unique point in $D$ at which $\mu(m, \theta)-0$, namely, m $-1-\theta$, $\theta=\underline{\theta}, \overline{\mathrm{m}}-\mathrm{R}(\underline{\theta})+1-\underline{\theta}$. If $(\mathrm{m}, \theta, \overline{\mathrm{m}})$ takes these values, and $\mathrm{m}^{\prime}>\mathrm{m}$, then $\mu\left(\mathrm{m}^{\prime}, \theta\right)>0, \mathrm{~J}\left(\mathrm{~m}^{\prime}, \theta, \overline{\mathrm{m}}\right)-\boldsymbol{l}, \mathrm{I}(0, \theta)-0$, so that $\mathrm{z}\left(\mathrm{m}^{\prime}, \theta, \overline{\mathrm{m}}\right)>0$ and $c\left(m^{\prime}, \theta, \bar{m}\right)>0$. But $c(m, \theta, \bar{m})=0$, so that $c\left(m^{\prime}, \theta, \bar{m}\right)>c(m, \theta, \bar{m})$. Thus $c(m, \theta, \tilde{m})$ is strictly increasing in $m$, on the entire domain $D$. Now let $f(m)$ be any function belonging to $\mathbf{F}^{\prime}$. It follows that $F(m, \theta, \bar{m})$ is strictly increasing in $m$, on the domain $D$. Because $\Gamma$ is increasing in $m$, it follows that $\phi(m, \theta)$ is strictly increasing in $m$, on the domain m $\geq 1-\theta$, $\theta \leq \theta \leq \bar{\theta}$. Finally, it follows from this that (If)(m) is strictly increasing in $m$, on the domain $m \geq 1-\ell$. Thus $T: F^{\prime} \rightarrow F^{\prime}$, and furthermore $f \in F^{\prime}$ implies that $T f$ is strictly increasing. Since $F^{\prime}$ is a closed subset of the complete metric space $F, T$ must have a fixed point in $F^{\prime}$, which is the same function $v(m)$ referred to above.

Furthermore, since $v=I v, v(m)$ must be monotonically increasing in $m$. and not merely nondecreasing.

(5) Finally, we show that $v(m)$ is concave. Let $F^{\prime \prime} \subset F$ be the se: of bounded, continuous functions that are also concave. We wish to show that $I$ maps $F^{\prime \prime}$ into itself as well.

We first show that the function $c(\mathbf{m}, \theta, \tilde{\mathbf{a}})$, defined above, is concave in $(m, \vec{m})$, for any $\theta \in \theta$. Let us define

$$
K(\mu, \theta, \overline{\mathbf{m}})=\theta \mu+R(\theta)+r(\theta) \cdot \overline{\mathrm{i}} .
$$

Then (A.1) can equivalently be written 


$$
I(z, \theta)-\frac{K}{\mu}
$$

which we can invert to obtain

$$
z-\bar{z}\left(\frac{8}{\mu}, \theta\right) \text {. }
$$

Because $S_{\pi}(c)$ is a non-decreasing function, $I(z, \theta)$ is a convex function of $z$, for any $\theta$. It follows that $\bar{z}\left(\frac{B}{\mu}, \theta\right)$ is a concave function of $\frac{K}{\mu}$. Then

$$
\bar{c}(K, \mu, \theta)=\mu \bar{z}\left(\frac{B}{\mu}, \theta\right)
$$

is a concave function of $(\mathrm{K}, \mu)$, for any $\theta$. But

$$
c(\mathbb{m}, \theta, \bar{m})-\bar{c}[K(\mu(m, \theta), \theta, \bar{m}), \mu(\bar{m}, \theta), \theta] .
$$

Since $K(\mu, \theta, \bar{m})$ is linear in $(\mu, \bar{m})$, and $\mu(m, \theta)$ is linear in $m$, for any $\theta$, it follows that $c(m, \theta, \overline{\mathrm{m}})$ is concave in $(\mathbf{m}, \overline{\mathbf{m}})$.

Now let $f(m)$ be any function belonging to $\mathbf{F}^{m}$. Then the concavity of $U(c)$ and $c(m, \theta, \bar{m})$ imply that $F(m, \theta, \bar{m})$ is concave in $(m, \bar{m})$, for any $\theta \in \theta$. Furthermore, for any $\theta \in \theta, D_{\theta}$ is convex, from which it follows that $\phi(m, \theta)$ is concave in $m$. As this is true for all $\theta$, the integral over $\theta$ must be concave in $m$ as well, so that (Tf)(II) is concave in $m$. Thus $T: F^{n} \rightarrow F^{n}$. As $F^{n}$ is a closed subset of the complete metric space $F, T$ must have a fixed point in $\mathbf{F}^{m}$, which must be the function $v$. Hence $v(m)$ is concave in m. $\square$ 
Proposition 4.2. In addition to the hypotheses of Proposition 4.1, suppose that

(1) the function $U(c)$ is continuously differentiable for all $c>$ 0 , it is strictly concave (1.e., $U^{\prime}(c)$ is monotonically decreasing), and $\lim _{c \rightarrow \infty} U^{\prime}(c)=+\infty$;

(i1) $S_{\pi}(c)<+\infty$ for some $c>0$; and

(iii) the function $c(\theta)$ that solves the maximization problem in (4.3c) for the case $w^{1}$ is such that (4.4) holds, and furthermore such that

$(4.7)$

$$
S_{\pi}(c(\theta)) \leq p(\theta)
$$

for all $\theta \in \theta$.

Then the value function $v(m)$ that satisfies (4.3a) is differentiable at $m=1$, and the derivative equals

$$
\mathrm{v}^{\prime}(1)-(1-\beta)^{-1} \int_{\theta}-1 \mathrm{U}^{\prime}(\mathrm{c}(\theta)) c(\theta)(\mathrm{d} \theta)
$$

Proof. (1) We first show that the function $c(\theta)$ is continuous, and bounded away from zero. The function $c(\theta)$ is defined as

(A.4a) $c(\theta)-\arg \max _{c} V(c, \theta)-U(c)+\beta v\left[1+R(\theta)-\int_{0}^{c} \min \left[S_{\pi}(x), p(\theta)\right] d x\right]$. subject to 
(A. 4b) $\max (1-1,1-\theta+R(\theta)) \leq 1+R(\theta)-\int_{0}^{c} \min \left[S_{\pi}(x), P(\theta)\right] d x \leq 1+R(\theta)$.

Because of (4.7), (A.4) is equivalent to the following definition.

(A.5a) $\quad c(\theta)-\arg \max _{c} \tilde{V}(c, \theta)=U(c)+\beta v\left[1+R(\theta)-\int_{0}^{c} S S_{\pi}(x) d x\right]$,

subject to

(A.5b) $\quad \max (1-\theta, 1-\theta+R(\theta)) \leq 1+R(\theta) \cdot \int_{0}^{c} S_{\pi}(x) d x \leq 1+R(\theta)$.

Condition (4.7) implies that $\overline{\mathrm{V}}(c, \theta) \leq \mathrm{V}(c, \theta)$ for all $c \geq 0, \theta \leq \theta \leq \bar{\theta}$, with $\bar{v}(c, \theta)-V(c, \theta)$ for all $c \leq c(\theta)$. Note also that $\bar{v}(c, \theta), 1$ ike $V(c, \theta)$, is a concave function of $c$, for all $\theta \leq \theta \leq \bar{\theta}$. Hence $c(\theta)$ is greater than, less than, or equal to the unconstralned maximizer of $V(c, \theta)$ only if it is respectively greater than, less than, or equal to the unconstrained maximizer of $\tilde{\mathrm{V}}(c, \theta)$. Furtherwore, each inequality in (A.4b) implies the corresponding inequality in (A.5b), and if either inequality is an equality, this implies the corresponding equality in ( $4.5 \mathrm{~b}$ ). Thus a solution to (A.4) is necessarily also a solution to (A.5). Finally, because of the strict concavity of $U(c)$ and the concavity of $v(m)$, the solution to (A.5) must be unique. Hence $c(\theta)$ is equivalently defined by (A.5). But the set of values for $c$ that satisfy (A.5b), considered as a function of $\theta$, constitute a continuous, compact-valued correspondence, while $\widetilde{V}(c, \theta)$ is seen to be a continuous function of both arguments (given 
the continulty of $v(m))$. Hence, by the theorem of the maximum, $c(\theta)$ is a continuous function.

We next observe that $c(\theta)>0$ for all $\theta$. For suppose instead that $c(\theta)-0$ for some $\theta \in \theta$. Then it follows from (4.4) that $R(\theta)-0$ as well. Then given that $\mathrm{s}_{\pi}(\mathrm{c})<+\infty$ for some $c>0$ (and hence for all lower values of $c$ as well), (A.Sb) is satisfied by all small enough $c>0$. Let $c^{*}>0$ be chosen so that (A.5b) is satisfied by all $0 \leq c \leq c^{*}$. Then the strict concavity of $U(c)$, the concavity of $v(m)$, and the convexity of $\int_{0}^{c} s_{\pi}(x) d x$ 1mply that

$$
\begin{aligned}
\bar{v}(c, \theta) & =U(c)+\beta v\left(1-\int_{0}^{c} S_{\pi}(x) d x\right) \\
& =U(0)+\beta v(1)+c\left(\frac{U(c)-U(0)}{c}-\frac{\theta}{c^{\star}}\left[v(1)-v\left(1-\int_{0}^{c *} S_{\pi}(x) d x\right)\right]\right) \\
& =\bar{v}(0, \theta)+c\left(\frac{U(c)-U(0)}{c} \cdot \frac{\beta}{c^{\star}}\left[v(1)-v\left(1-\int_{0}^{c *} S_{\pi}(x) d x\right)\right]\right) .
\end{aligned}
$$

Because $U^{\prime}(c)$ becomes unboundedly large as $c$ approaches zero, the expression in curly brackets must be positive for small enough $c>0$. Hence $\bar{V}(c, \theta)$ is not maximized at $c-0$, a contradiction. Then since $c(\theta)$ is a continuous function on the compact set $\theta$, it is not only everywhere positive, but bounded away from zero.

(2) Now for any $m_{0} \geq 1-\phi$, and any sequence of monetary infections $\theta$ - $\left(\theta_{t}\right)$, let sequences $\left(\bar{c}_{t}\left(\mathrm{~m}_{0} ; \theta\right), \overline{\mathrm{m}}_{t}\left(\mathrm{~m}_{0} ; \theta\right)\right)$ be defined recursively by the relations 


$$
\begin{aligned}
& \overline{\mathrm{m}}_{0}\left(\mathrm{~m}_{0} ; \theta\right)-\mathrm{m}_{0}, \\
& \tilde{\mathrm{m}}_{t+1}\left(\mathrm{E}_{0} ; \theta\right)=\mu\left(\overline{\mathrm{I}}_{\mathrm{t}}\left(\mathrm{m}_{0} ; \theta\right), \theta_{\mathrm{t}}\right)\left[1-\mathrm{R}\left(\theta_{\mathrm{t}}\right) \cdot+\left(\theta_{\mathrm{t}}\right)\right]+\mathrm{R}\left(\theta_{\mathrm{t}}\right)++\left(\theta_{\mathrm{t}}\right), \\
& \bar{c}_{t}\left(\mathbb{I}_{0} ; \theta\right)-\mu\left(\tilde{\mathbb{I}}_{t}\left(\mathbb{m}_{0} ; \theta\right), \theta t\right) c(\theta) .
\end{aligned}
$$

Note that for any $m_{0}$ and any sequence of monetary infections, the sequences $\left(\tilde{c}_{t}\left(\mathbb{m}_{0} ; \theta\right), \overline{\mathbb{m}}_{t}\left(\mathbb{m}_{0} ; \theta\right)\right\}$ represent a plan that satisfies (4.3b)(4.3d) in all periods. Furthermore, when $\mathrm{m}_{0}=1$, this is the optimal plan, namely $\bar{c}_{t}-c\left(\theta_{t}\right), \overline{\mathrm{m}}_{t+1}-1$ for all $t$. Then let $\vec{v}\left(\mathbb{m}_{0}\right)$ denote the level of utility obtafned under this plan, i.e..

$$
\tilde{v}\left(\mathrm{~m}_{0}\right)=E\left(\sum_{t=0}^{\infty} \beta \bar{t}\left(\tilde{c}_{t}\left(\mathrm{~m}_{0} ; \beta\right)\right)\right\}
$$

where the expectation is over the different possible histories of wonetary injections $\theta$. It is easily seen that for each $t, \tilde{c}_{t}\left(\mathbb{m}_{0} ; \theta\right)$ is a continuous function of $\theta$, so that $\mathrm{U}\left(\tilde{c}_{t}\left(\mathrm{~m}_{0} ; \theta\right)\right)$ is a bounded continuous function, with the same bounds for all $t$. Hence the integral involved in the above definition is well-defined for each $t$, and the uniform bounds imply that the infinite sum of integrals must converge. Hence $\tilde{v}\left(m_{0}\right)$ is well-defined for each $\mathrm{m}_{0} \geq 1-\phi$.

We wish to show that $\bar{v}\left(\mathbb{m}_{0}\right)$ is differentiable at $m_{0}-1$. For $\epsilon \geq-\ell, \epsilon \neq 0$, let us define

$$
\rho(\epsilon)=\frac{\bar{v}(1+\epsilon)-\bar{v}(1)}{\epsilon} .
$$


Then we wish to show that the function $f(c)$ is continuous at $t=0$; the limiting value $\rho(0)$ is then $\bar{v}^{\prime}(1)$. From the definition of the processes $\left(\tilde{c}_{c}, \tilde{m}_{t}\right)$, it is obvious that

$$
\begin{aligned}
\vec{v}\left(m_{0}\right)-E\left(U\left(\mu\left(m_{0}, \theta_{0}\right) c\left(\theta_{0}\right)\right)\right. \\
\\
\left.\quad+\beta \bar{v}\left(\mu\left(m_{0}, \theta_{0}\right)\left[1-R\left(\theta_{0}\right)-+\left(\theta_{0}\right)\right]+R\left(\theta_{0}\right)++\left(\theta_{0}\right)\right)\right\}
\end{aligned}
$$

where here the expectation is over the possible realizations of $\theta_{0}$. This implies that for any $c \geq-1$, $c+0$,

$$
\rho(\epsilon)-\int[u(\epsilon, \theta)+\beta \rho(\lambda(\theta) \epsilon)] \Phi(d \theta)
$$

where

$$
\begin{aligned}
\lambda(\theta) & =\frac{1-R(\theta)-+(\theta)}{\theta}, \\
u(c, \theta) & =\frac{1}{\epsilon}\left[U\left(\frac{c+\theta}{\theta} c(\theta)\right)-U(c(\theta))\right] .
\end{aligned}
$$

Note that $(4.3 b)-(4.3 c)$, together with (4.4), imply that $0 \leq R(\theta) \leq \theta$ for all $\theta$, so that $0 \leq \lambda(\theta) \leq 1$ for all $\theta$. And the facts that $U(c)$ is continuously differentiable for all $c>0$, and that $c(\theta)$ is a continuous function bounded away from zero, Imply that if we adjoin to the above definition the stipulation

$$
u(0, \theta)=\frac{c(\theta) u^{\prime}(c(\theta))}{\theta}
$$


then $u(\epsilon, \theta)$ is a continuous function on the domain $\in \geq-\theta, \theta \leq \theta \leq \bar{b}$. Finaliy the concavity of $U(c)$ implies that for any $\theta \leq \theta \leq \bar{\theta}$,

$$
0 \leq u(\theta, \theta) \leq \frac{U(c(\theta))-U(0)}{\theta}
$$

for all $\epsilon \geq-\theta$. As the right hand expression is a bounded function of $\theta$, it follows that $u(\epsilon, \theta)$ is a bounded function.

Now for any bounded continuous function $f(\epsilon)$ on the domain $\epsilon \geq-\phi$, let
(A.7)
(Tf) $(\epsilon)-\int[u(\epsilon, \theta)+\beta f(\lambda(\theta) \epsilon)] \Phi(d \theta)$.

From the properties just mentioned, it is evident that $u(\epsilon, \theta)+\beta f(\lambda(\theta) \epsilon)$ is well-defined for all $\varepsilon \geq \cdots, \theta \in \theta_{i}$ and that lt is furthermore a bounded continuous function on this domain. It then follows that (Tf) ( $\theta$ ) is well-defined for all $\epsilon \geq-\ell$, and furthermore a bounded continuous function of $\epsilon$ on this domain. Thus (A.7) defines an operator $T$ that maps the set of bounded concinuous functions on the domain $[-f,+\infty)$ into itself. As it satisfies the Blackwell conditlons (with the sup norm), it is a contraction, and so has a unique fixed point. Comparison of (A.7) with (A.6) shows that the fixed point coincides with the function $\rho(c)$ defined earlier, for all $\epsilon \neq 0$. Since the fixed point is a continuous function, $\rho(\epsilon)$ is continuous at $\epsilon-0$. Thus $\tilde{v}(m)$ is differentiable at $m-1$, and the derivative is $\tilde{v}^{\prime}(1)-\rho(0)$.

From (A.7) we observe that the fixed point, evaluated at $\epsilon=0$, must satisfy 
63

$$
\rho(0)=\int[u(0, \theta)+\beta \rho(0)] \Phi(d \theta)
$$

so that

$$
\bar{v}^{\prime}(1)=(1-\beta)^{-1} \int_{\theta}^{-1} U^{\prime}(c(\theta)) c(\theta)(d \theta) .
$$

(3) Since $\left(\vec{c}_{t}\left(m_{0} ; \theta\right), \vec{m}_{t}\left(m_{0} ; \theta\right)\right)$ represents a feasible plan, it follows that $\tilde{v}(m) \leq v(m)$ for all m $\geq 1-\theta$. Furthermore, $\tilde{v}(1)-v(1)$. One also observes that $\vec{c}_{t}\left(m_{0} ; \theta\right)$ is a linear function of $m_{0}$, for each $\theta$, as a result of which $\tilde{v}(m)$ is a concave function of $m$. Then the fact that $\vec{v}(m)$ is differentiable at $w-1$ implies that $v(m)$ is differentiable at that point as well, by the lemma of Benveniste and Scheinkman (Stoked, Lucas, and Prescott, Theorem 4.10), and that the derivative is $v^{\prime}(1)$ $\vec{v}^{\prime}(1)$. Then (A.8) implies (4.8). 


\section{Foetnotes}

1 In the symetric equilibria that we characterize below, each player of a given type chooses the same quantity, so that the integrals exist (and equal the common quantity chosen). When an individual player considers choosing a different quantity, he expects to have no effect on the value of the integral.

${ }^{2}$ Given the strategies of the other players and $i$ 's choices in the earliet stages, the history $\left(c_{11}, \ldots, c_{k-1,1}\right)$ is also a function of $\theta$. Hence we could simply have consumer 1 choose a sequence of functions $x_{k i}(\theta)$. But In this case we would not be able to define i's strategy set independently of the strategies chosen by the other players. The definition used here allows us to define each player's strategy set independently, and to define a payoff function on the product of these strategy sets.

3 We describe the producer's strategy as a measure rather than a sequence in order to suggest the generalization to the case of a continuous price set, treated below.

4 We assume furthermore that even if "all sales are public, "this only means that the functions $z_{k j}$ and $c_{k i}$ are revealed up to the equivalence class of almost everywhere identical functions (with respect to Lebesgue measure) to which these functions belong. Thus an individual player who chooses to deviate from equilibrium play does not expect that other players can observe his action, and cannot expect their strategies to respond to it. ${ }^{5}$ Note that in this model, beyond a certain point a higher realization of the money supply has no effect on elther prices or real activity. The additional money is simply hoarded. This kind of "liquidity trap" is a typical feature of cash-in-advance models, and has nothing to do with the 
special market structure proposed here. See, for example, the discussion of the corresponding equilibrium with Walrasian spot markets in the next paragraph.

${ }^{6}$ of course, real effects of anticipated money growth are possible in such models, if there are possibilities for substituting away from cash transactions. Such effects are entrely due to the fact that higher anticipated inflation increases the cost of using cash, and so increases the resort to inefficient alternatives. In this paper, we abstract entirely from this source of monetary non-neutrality.

7 We do not consider here whether average output is monotonic in some measure of the degree of uncertainty. In real1ty, of course, capacity production is determined by cholce, not given as we assume here, and calculating the response of average equilibrium output to changes in the level of uncertainty would require taking the cholce of capacity into account as well as the extent to which existing capacity is utilized.

${ }^{8}$ The predicted procyclical movements in production are even greater if one allows for the use of intermediate goods in production, as shown by Basu (1992).

9 Eden's model differs from ours in that successive monetary injections are recelved by different consumers, but this has little consequence for the real effects of monetary shocks in his model. In particular, the conditions for optimal pricing by producers are unaffacted by this change. 


\section{Ist of References}

Basu, Susanto. 1992. "Intermediate Goods, Menu Costs, and Business Cycles." Harvard University working paper.

Blanchard, Olivier J., and Nobuhiro Kiyotaki. 1987. "Monopolistic Competition and the Effects of Aggregate Demand." American Economic Review 77: $647-666$.

Butters, Gerard. 1977. "Equilibrium Distributions of Sales and Advertising Prices, "Revlew of Economic Studies 44:467-491.

Eden, Benfamin, 1990a, Marginal Cost Pricing when Spot Markets are Complete." Journal of Polltical Economy 98:1293-1306. 1990b. "Complete Spot Markets and the Non-Neutrality of Money." University of Iowa working paper. 1991. "The Adjustment of Prices to Monetary Shocks when Spot Markets are Complete." University of Iowa working paper. Friedman, Milton, and Anna J. Schwartz. 1963. A Wenetary History of the United States, 1867-1960. Princeton: Princeton University Press. Hall, Robert E. 1988. "The Relation Between Price and Marginal Cost in U.S. Industry." Journal of Political Economy $96: 921-947$. 1990. "Invariance Properties of Solow's Productivity Residuals." in P.A. Diamond, ed., Growth/Productivity/Unemployment. Cambridge: M.I.T. Press.

King, Robert G. 1991. "Money and Business Cycles." University of Rochester working paper.

Lucas, Robert E., Jr, 1972. "Expectations and the Neutrality of Money." Journal of Economic Theory 4;103-124. 
Prescott, Edward C. 1975. "Efficlency of the Natural Rate." Leurnal of Political Economy. 83: $1229-1236$.

Rotemberg, Jullo J. 1988. "Rationing in Centrally Planned Economles." M.I.T. working paper. and Lawrence H. Summers. 1990. "Inflexible Priceg and Labor Hoarding's Role In Explainfing Procycical Productivity." Quarterly. Journal of Economics 105:851-874.

Sargent, Thomas J. 1987. Dynamic Macroeconomic Theory. Cambridge: Harvard Unfversity Press.

Stokey, Nancy L., Robert E. Lucas, Jr, and Edward C. Prescott. 1989. Recursive Methods in Economic Dynamics. Canbridge: Harvard Unfversity Press.

Svensson, Lars E.O. 1986. "Sticky Goods Prices, Flextble Asset Prices, Monopolistic Competition, and Monetary Policy." Review of Economic Studies 53:385-405. 


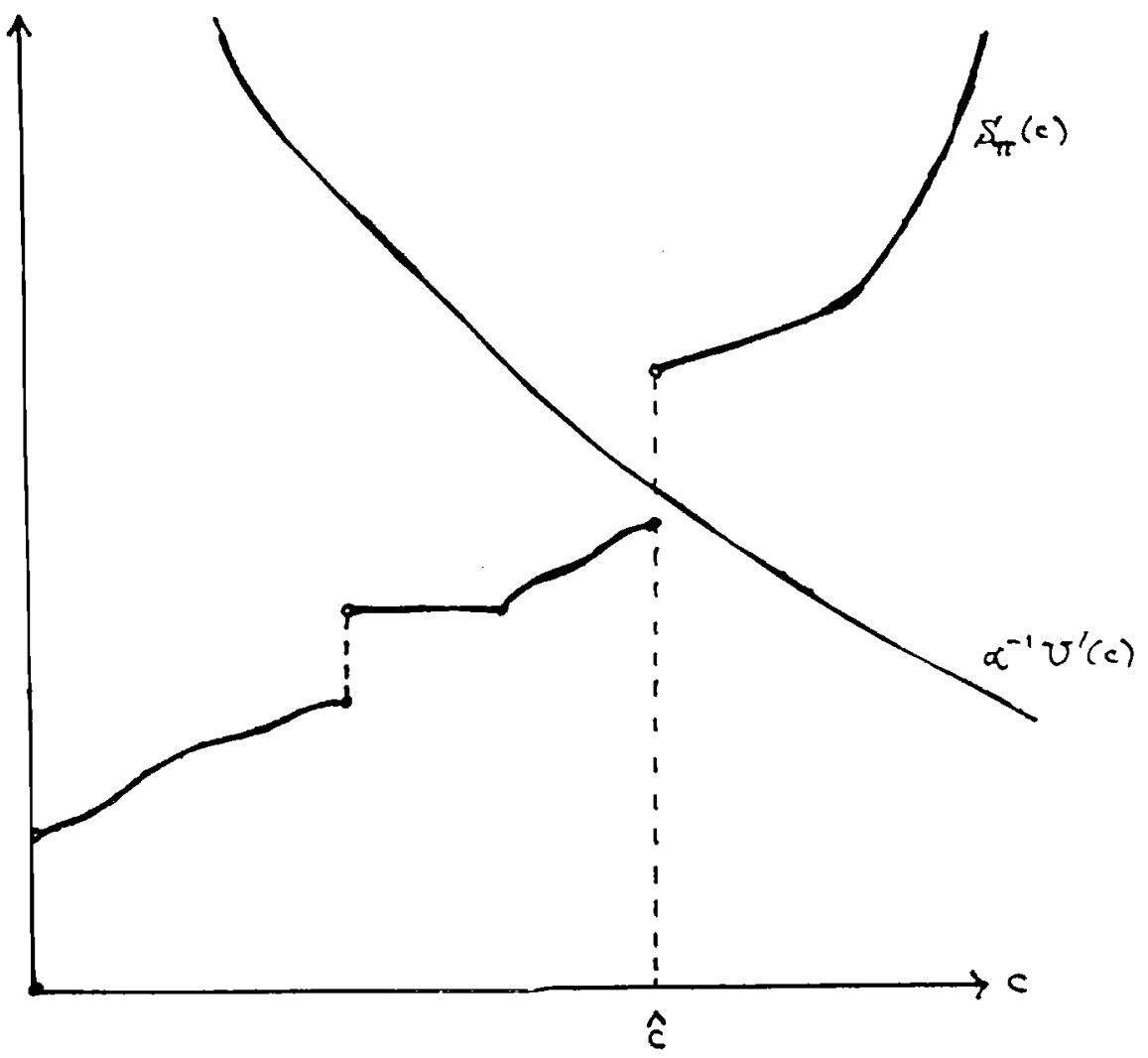

Figure 1. 